\title{
TRAYECTORIAS EDUCACIONALES E INSERCIÓN LABORAL EN LA ENSEÑANZA MEDIA TÉCNICO PROFESIONAL
}

\author{
Osvaldo Larrañaga \\ Programa de las Naciones Unidas para el Desarrollo \\ Gustavo Cabezas \\ Programa de las Naciones Unidas para el Desarrollo
}

Francisca Dussaillant

Universidad del Desarrollo

\begin{abstract}
Osvaldo Larrañaga. Economista de la Universidad de Chile y doctor en economía de la Universidad de Pennsylvania. Actualmente se desempeña como encargado del área de pobreza y desigualdad de la oficina del Programa de las Naciones Unidas para el Desarrollo (PNUD) en Chile. Email: osvaldo.larranaga@undp.org.

Gustavo Cabezas. Economista y magíster en economía aplicada de la Universidad Alberto Hurtado. Trabaja en el área de pobreza y desigualdad del PNUD en Chile. Email: gustavo.cabezas@undp.org.

Francisca Dussaillant. Ingeniera civil industrial de la Universidad Católica. Master en educación en la Universidad de Carolina del Norte y doctora en economía de la Universidad Católica. Actualmente está afiliada a la Facultad de Gobierno de la Universidad del Desarrollo. Email: mfdussaillant@udd.cl.

Este artículo está basado en un estudio que el Ministerio de Educación de Chile encargó al PNUD. El informe completo del estudio puede ser consultado en la página web del PNUD (Larrañaga, Cabezas \& Dussaillant 2013). El Mineduc proveyó los datos que sustentan la investigación y se cuenta con su aprobación para realizar la presente publicación. Este artículo, sin embargo, es exclusiva responsabilidad de los autores y no representa la opinión del PNUD ni demás agencias del Sistema de Naciones Unidas. Los autores agradecen los comentarios de los dos árbitros de la revista Estudios Públicos, así como aquellos formulados por Claudia Peirano, Paulina Araneda, Hernán Araneda, Celia Alvariño, Carlos Henríquez, Rodrigo Herrera, Denise Falck y de profesionales del PNUD a versiones preliminares del documento. Asimismo, el estudio base que origina el documento contó con los comentarios de Francisco Lagos, Gabriel Ugarte, Alejandro Weinstein, Loreto Cox y otros profesionales del Mineduc.
\end{abstract}


RESUMEN: Alrededor del 45 por ciento de los estudiantes que se gradúan en la enseñanza media en Chile cursan la educación técnico profesional. El presente estudio entrega evidencia empírica sobre sus trayectorias educacionales y su inserción laboral. Para ello se sigue a través de registros administrativos a toda la cohorte de estudiantes que cursó segundo medio en el año 2003, a través de su paso por la enseñanza media, educación superior e inserción laboral. El documento cubre la elección de la modalidad de enseñanza media técnico profesional versus científico humanista, los resultados de aprendizaje en el Simce de segundo medio y en la Prueba de Selección Universitaria, la deserción en la enseñanza media, el acceso a la educación terciaria por tipo de institución, la graduación versus deserción en los estudios terciarios, la inserción en el mercado laboral y las remuneraciones obtenidas.

PALABRAS ClAVE: educación media técnico profesional, trayectorias educacionales, inserción laboral, diferencias salariales de género. RECIBIDO: septiembre 2013; ACEPTADO: abril 2014.

\title{
EDUCATIONAL PATH AND LABOR MARKET INSERTION IN SECONDARY VOCATIONAL SCHOOLS
}

\begin{abstract}
Secondary vocational educational represents around $45 \%$ of students who graduate from secondary schools in Chile. This study provides empirical evidence about the educational trajectories and labor market insertion of the complete cohort of students that attended 10th grade in the year 2003. We use administrative data which allows for following individual students through secondary education, higher education and into the labor market. The paper addresses the choice between vocational and general secondary education, learning outcomes in the 10th grade Simce Standardized Test and the University Entry Test, the dropout rate in secondary education, entry to higher education, the dropout versus graduation rates in higher education institutions, labor market participation and labor earnings.
\end{abstract}

Keywords: Secondary vocational education, educational trajectories, labor market insertion, gender pay gap.

ReCEIVED: September 2013; ACCEPTED: April 2014.

\section{INTRODUCCIÓN}

La educación media técnico profesional congrega actualmente a un gran número de estudiantes en el país: entre el 40 y 45 por ciento de los alumnos de tercero y cuarto de enseñanza media, dependiendo de la cohorte. Este porcentaje aumenta a alrededor del 60 por ciento entre los estudiantes de los tres primeros quintiles de ingreso. 
De acuerdo con el marco curricular que introduce la reforma de 1998, la formación media técnico profesional "ofrecerá a los alumnos y las alumnas oportunidades de realizar aprendizajes en un campo de especialización que facilite su acceso a un primer trabajo remunerado, atendiendo a sus intereses, aptitudes y disposiciones vocacionales, mediante una formación técnica en el ámbito de un sector del mundo productivo, que los prepare en forma efectiva para el trabajo y para responder con flexibilidad a la velocidad de los cambios tecnológicos". ${ }^{1}$

Así, el objetivo de la educación media técnico profesional es formar competencias para el trabajo y debiera ser un pilar importante de la formación de capital humano del país. También debiera contribuir a la movilidad social en vista de la composición socioeconómica de sus estudiantes. Sin embargo, la enseñanza media técnico profesional ha estado relativamente ausente del debate educacional de los últimos años, posiblemente porque la composición socioeconómica de sus alumnos la convierte en un sector educacional con baja visibilidad política.

A la falta de visibilidad del sector contribuye también la carencia de estudios e indicadores que informen sobre los resultados que obtienen los estudiantes en el campo educacional y laboral. Así, existe un conjunto de preguntas abiertas sobre el futuro de la enseñanza media técnico profesional: ¿está respondiendo a los objetivos que plantea su marco curricular y preparando a los estudiantes para insertarse en el mercado del trabajo? ¿Sigue siendo válida como una fase terminal de estudios o debe también preparar a sus alumnos para que continúen los estudios en el nivel terciario? ¿Hay que dar mayor peso en el currículo a los conocimientos y habilidades generales para facilitar los ajustes posteriores en el mercado del trabajo?

El presente estudio tiene por objetivo proveer evidencia empírica que contribuya a dar respuesta a estas preguntas y otras relacionadas. Para ello, se analizan las trayectorias educacionales y la inserción laboral de toda la cohorte de estudiantes que cursó segundo medio en el año 2003, utilizando para tal efecto registros administrativos individuales de los estudiantes durante su paso por la enseñanza media, la enseñanza superior y su inserción en el mercado laboral. Esta cohorte fue elegida porque es la que permite el mejor aprovechamiento de los datos disponibles.

${ }^{1}$ Ministerio de Educación (Mineduc) de Chile, 2005. Este nuevo marco curricular empieza a implementarse en el año 2001. 
El análisis cubre los principales hitos de las trayectorias seguidas por estos jóvenes: ingreso a la educación media técnico profesional o científico humanista, resultados de aprendizajes en el Simce de segundo medio y en la Prueba de Selección Universitaria, deserción en la enseñanza media, acceso a la educación terciaria por tipo de institución, graduación versus deserción de los estudios terciarios, inserción en el mercado laboral y remuneraciones obtenidas.

El estudio es inédito en términos de las bases de datos utilizadas, puesto que investigaciones previas en la materia no dispusieron del conjunto de fuentes aquí disponibles. Una excepción es el estudio de Ministerio de Educación (2011) en tanto accede a las mismas bases de datos, pero su análisis refiere a un período bastante más corto (cohorte del año 2008 seguida en los años 2009 y 2010).

El estudio entrega una visión comprehensiva de las trayectorias seguidas por los alumnos de la cohorte, pero cada uno de los temas tratados puede ser objeto de investigaciones más específicas y detalladas. En particular, el presente trabajo no tiene por objetivo la estimación de efectos causales, los cuales requieren del uso de métodos experimentales o la aplicación de técnicas estadísticas mediante instrumentos que provean variación exógena en la variable de interés. Por regla general, es difícil identificar efectos causales y ello excede el ámbito del estudio. Se sigue que los resultados aquí obtenidos no pueden ser interpretados como causados por la enseñanza técnico profesional, según se discute en el texto correspondiente.

Asimismo, los resultados del estudio no reflejan a la educación técnico profesional en abstracto, sino como parte del sistema que operaba en Chile en la década que va de 2000 a 2010. Éste se origina en la reforma curricular de fines de los años 90, que recopiló un conjunto de antecedentes sobre las competencias laborales que demandaba el mercado del trabajo y que pasaron a formar parte del currículo de enseñanza (Miranda 2003). Sin embargo, las evaluaciones más recientes muestran que alrededor de la mitad de los módulos de formación no se completan porque requieren más tiempo que el disponible en el año escolar (Espinoza 2011). Igualmente, hay falta de equipamiento adecuado en los establecimientos y escasez de profesores que posean las calificaciones necesarias para la enseñanza (Ministerio de Educación 2011).

El documento se organiza en cuatro secciones. En primer término, se presentan antecedentes generales sobre la educación media técnico profe- 
sional y se describen las bases de datos usadas en el estudio; en segundo lugar, se presentan los resultados de las trayectorias educacionales de la cohorte bajo análisis; la tercera sección presenta la inserción laboral de la cohorte, y en la última sección se sintetizan los principales resultados obtenidos.

\section{ANTECEDENTES Y DATOS}

\subsection{Antecedentes}

No hay un modelo único en el mundo en términos de la organización de la educación secundaria. La mayoría de los países de Europa y América Latina, Chile incluido, organizan la educación media en modalidades alternativas de enseñanza general y vocacional (técnico profesional en Chile), aunque hay diferencias en la etapa del ciclo educacional en que éstas se separan, en la existencia o no de establecimientos exclusivos para cada modalidad, en el número de especialidades de la educación técnica, así como en otras materias. Algunos países ofrecen formación vocacional de tipo dual, en forma alternada en las escuelas y lugares de trabajo (Alemania, Austria y Suiza, entre otros). También hay casos, como Estados Unidos y Nueva Zelandia, en que todos los alumnos son cubiertos por un currículo común de educación general, si bien pueden cursar asignaturas opcionales de contenido vocacional.

La proporción de estudiantes en educación vocacional es también variable. Dentro de los países de la OECD, ella fluctúa entre el 65 y 80 por ciento en Europa central; en torno al 50 por ciento en los países escandinavos; entre el 30 y 40 por ciento en Europa mediterránea, Japón y Corea, y es prácticamente inexistente en Estados Unidos y Nueva Zelandia (Sahlberg 2007).

Las variantes de organización de la enseñanza secundaria responden por lo general a desarrollos históricos de los países, no obstante la enseñanza vocacional congrega a la mitad o más de los estudiantes en la mayoría de los países desarrollados. Hay, eso sí, una tendencia a reducir el nivel de especialización en la formación vocacional, habida cuenta de la cambiante realidad del mercado laboral, que exige de competencias generales por parte de los trabajadores para adaptarse mejor a nuevos empleos. También son notorios los esfuerzos que están realizando los países desarrollados para 
implementar estrategias de formación de competencias laborales a lo largo del ciclo de vida de los trabajadores (OECD 2009).

La estructura de la educación escolar vigente proviene básicamente de la reforma educacional de Frei Montalva en 1965, que extendió la educación primaria de seis años de "preparatorias" a ocho años de educación básica y creó la educación media de cuatro años en modalidades de enseñanza general y técnico profesional. Previamente, la educación secundaria consistía en seis años de educación general de "humanidades", que acogían a un porcentaje minoritario de jóvenes que luego cursaban estudios superiores o se integraban al mercado laboral en ocupaciones de oficinas. También había establecimientos de enseñanza técnica en el ámbito comercial y de oficios, incluida la formación de profesores primarios en las escuelas normales.

Bajo el currículo vigente desde 1998, los estudiantes de educación media cursan los dos primeros años en educación general y los dos últimos, en modalidad diferenciada, si bien en la práctica la elección entre modalidades ocurre al inicio de la enseñanza media, dado que la mayoría de los establecimientos a partir de tercero medio ofrece sólo un tipo de enseñanza u otra. ${ }^{2}$ La modalidad técnico profesional contiene un conjunto de opciones de especialización agrupadas en sectores económicos y los egresados que completan una práctica profesional reciben el título de técnico de nivel medio.

\subsection{Revisión de la literatura}

La literatura internacional contiene una gran diversidad de investigaciones respecto de la educación secundaria vocacional. En materia del desempeño laboral, la mayor parte de los estudios realiza una evaluación comparativa entre los egresados de la educación secundaria vocacional versus general, con resultados que varían según la población evaluada y los datos y metodologías utilizados. El artículo de Meer (2005) provee una revisión útil de esta literatura. Una dificultad común en la estimación de los retornos de la educación vocacional es separar el efecto de la enseñanza respecto de atributos no observados que hacen distintos a los estudiantes en educación vocacional versus general (Malamud \& Pop-Eleches 2008). También es relevante el horizonte de evaluación, dado que las competencias específicas adquiridas en la enseñanza vocacional pueden ser más efectivas en los

${ }^{2}$ En el año 2009, había sólo 19 por ciento de establecimientos de enseñanza media que ofrecían ambas modalidades de estudios (establecimientos polivalentes). 
primeros años de la inserción laboral, mientras que en el mediano y largo plazo adquieren más valoración las competencias generales que faciliten la adaptación a nuevos trabajos (Hanushek, Woessmann \& Zhang 2011).

Otra línea de investigaciones refiere al efecto que sobre la igualdad de oportunidades tiene la asignación de alumnos en modalidades diferenciadas de enseñanza, dado que la mayoría de los estudiantes de familias vulnerables asiste a educación vocacional. Las investigaciones comparan países que difieren en el número de años en que los alumnos se ven expuestos a separación en las modalidades general y vocacional, o bien analizan situaciones de cambio de regímenes al interior de países. Los resultados muestran que mientras menos expuestos están los alumnos a contenidos de formación general, mayor tiende a ser la incidencia del nivel socioeconómico sobre los resultados, deteriorándose la distribución de oportunidades (Brunello \& Checchi 2007).

A nivel internacional, la literatura muestra que los estudiantes de la enseñanza vocacional obtienen en promedio resultados educacionales inferiores en las pruebas TIMSS y PISA. Los resultados más bajos de la educación vocacional pueden deberse a razones intrínsecas de esta modalidad — es un currículo más acotado en asignaturas de formación general-, pero también podrían dar cuenta de una población de estudiantes con menores niveles de habilidades iniciales o profesores de menor calidad. Sin embargo, hay autores que postulan que la educación vocacional contribuye a la formación de rasgos de carácter, que derivan en mejores resultados educacionales en los grupos vulnerables. Una revisión de esta literatura se encuentra en Farías (2013).

En Chile ha existido un conjunto de estudios basados en microdatos que evalúan la inserción laboral de los egresados de la educación media técnico profesional en relación con los egresados de la modalidad científico humanista. Una primera ronda de estudios está basada en encuestas realizadas a muestras de egresados de ambas modalidades, cuyos resultados muestran que los egresados de la enseñanza técnico profesional tienen tasas más altas de ocupación y salarios iguales o mayores al grupo de comparación. Las evaluaciones refieren a los primeros años de la inserción laboral y no controlan por sesgos de selección en atributos no observables de los egresados. Ver en particular Cáceres \& Bobenrieth (1993), Bravo (1999) y Centro de Microdatos (2009).

Una segunda ronda de estudios utilizan microdatos de carácter censal provenientes de registros administrativos, como en el caso del Ministerio 
de Educación (2011) y Bucarey \& Urzúa (2013a). Estos últimos autores utilizan variables instrumentales para corregir por el efecto de selección en la distribución de los estudiantes entre las modalidades de enseñanza media, para encontrar que los jóvenes que egresan de la educación media técnico profesional obtienen una menor remuneración promedio que aquellos que egresan de la enseñanza científico humanista, pero el resultado varía de acuerdo con las ramas de estudio en la enseñanza vocacional.

Otras investigaciones también basadas en microdatos han estudiado la elección entre educación media técnico profesional y científico humanista. Farías (2013) releva el rol que juegan los factores culturales y muestra que el entorno sociocultural empuja a estudiantes vulnerables con potencial académico a estudiar educación media técnico profesional, una elección que los situaría en desventaja para seguir estudios universitarios. ${ }^{3}$ Ver también, Cáceres \& Bobenrieth (1994), De Iruarrizaga (2009) y Centro de Microdatos (2009).

Otro trabajo relevante es Bassi et al. (2012), donde se muestra que los egresados de la educación media técnico profesional contarían con una mayor dotación de habilidades socio-emocionales que sus pares de la enseñanza científico humanista, y que tal resultado se originaría en los hábitos que forma la enseñanza aplicada al trabajo.

Información también pertinente es provista por estudios cualitativos que indagan sobre las aspiraciones y expectativas de los estudiantes de la educación media técnico profesional. Sepúlveda et al. (2010) muestran que la mayoría de los alumnos del sector proyectan continuar sus estudios en el nivel superior y que para ellos la enseñanza media técnico profesional es un recurso que les permite tener un empleo a su egreso del nivel y así poder financiar el costo directo e indirecto de los estudios superiores. Evidencia en tal sentido es provista también por Ortiz (2009).

\subsection{Los datos y la cohorte de análisis}

El estudio analiza las trayectorias educacionales y la inserción laboral de la cohorte que cursó segundo medio en el año 2003. Ello se realiza siguiendo a la cohorte a través de microdatos contenidos en registros ad-

${ }^{3}$ Esta referencia corresponde a la tesis doctoral del autor, la que fuera desarrollada en paralelo al presente trabajo. Hay algunos temas que se superponen entre ambos documentos con resultados por lo general coincidentes, como se hace notar a lo largo de este documento. 
ministrativos, que se unifican a través del Registro Único Nacional de cada estudiante. Como resultado se obtiene una base de datos longitudinal, que cubre las trayectorias de la cohorte en la educación media y superior, así como en el mercado laboral, en el período que va desde 2003 a 2012.

Las bases de datos utilizadas son las siguientes: ${ }^{4}$

- $\quad$ Registro de Estudiantes de Chile (RECh), años 2003 a 2011. Contiene datos longitudinales anuales para todos los estudiantes en el ciclo de la enseñanza básica y media. Permite conocer el establecimiento al que asiste el estudiante, el grado de estudios y la modalidad de enseñanza, entre otras variables.

- $\quad$ Servicio de Información de la Educación Superior (SIES), años 2007 a 2011. Contiene datos longitudinales anuales para la casi totalidad de los estudiantes en educación superior. ${ }^{5}$ Permite conocer el tipo de estudios, el establecimiento y la carrera cursada, entre otras variables.

- Simce 2003 de segundo medio. Informa sobre las características de los hogares y los resultados obtenidos en la prueba Simce de ese año.

- Pruebas de Selección Universitaria de los años 2006 en adelante. Informa sobre los resultados obtenidos en la PSU y las características de los estudiantes y sus hogares.

- Base de datos del Seguro de Cesantía, año 2003 a 2012. Contiene datos longitudinales mensuales de los asalariados que cotizan en el Seguro de Cesantía, que son todos aquellos que tienen contrato de trabajo con excepción de los empleados públicos.

Para la cohorte principal no se dispone de mediciones de logro académico previo a la enseñanza media, puesto que a ella no le correspondió por calendario rendir el Simce de cuarto u octavo básico. Para relacionar el

${ }^{4}$ Las bases de datos empleadas en el estudio son propiedad del Ministerio de Educación y del Ministerio del Trabajo y Previsión Social. Estas bases pueden ser solicitadas por investigadores interesados a las respectivas instituciones. No obstante, la vinculación de los distintos microdatos se realiza a través del Registro Único Nacional de cada estudiante, información que no es pública. La base de datos unificada que dispuso esta investigación fue creada especialmente para estos efectos por el Ministerio de Educación con el concurso del Ministerio del Trabajo. Esta base se encuentra en poder de la primera entidad y puede en principio ser solicitada para otras investigaciones. Asimismo, es posible actualizar esta base de datos con registros administrativos más recientes, ampliando el período de análisis para los miembros de la cohorte.

${ }^{5}$ En el año de inicio de la base (2007) se incluyen 71 de un total de 72 centros de formación técnica, 35 de 38 institutos profesionales y 56 de 57 universidades. 
logro en la educación básica con las trayectorias educacionales posteriores se utilizan, a modo de complemento, los datos de una segunda cohorte, denominada en adelante "cohorte auxiliar", correspondiente a los alumnos que rindieron el Simce de octavo básico en 2004. Las características de los alumnos de esta segunda cohorte son muy similares a los alumnos de la cohorte principal, por lo que la información allí provista debiera aproximar razonablemente bien las tendencias de la cohorte principal.

La cohorte principal de análisis contiene 106.562 estudiantes en la modalidad técnico profesional (TP) y 129.157 en la modalidad científico humanista $(\mathrm{CH}) ; 45,2$ y 54,8 por ciento, respectivamente. ${ }^{6}$ Los hombres tienen mayor representación en la modalidad TP, puesto que el 47,8 por ciento de ellos sigue esta modalidad, comparado con el 42,6 de las mujeres.

La figura 1 describe en una línea de tiempo la cobertura de las bases de datos disponibles para la cohorte principal. Hay que notar que esta cohorte egresa oportunamente de la enseñanza media en 2005 y que el SIES registra los datos de los estudiantes en la educación superior desde el año 2007. Por tanto, hay un "punto ciego" en el año 2006, cuyo efecto práctico es no poder identificar a quienes accedieron $y$ desertaron de la educación superior en ese año. ${ }^{7}$ En cambio, no hay problemas para identificar a quienes ingresaron a la educación superior ese año y siguieron estudiando en años posteriores, puesto que ellos quedan registrados en el SIES a partir del 2007.

Los datos laborales provienen de la base del Seguro de Cesantía. Esta base de datos registra mes a mes la remuneración y otra información del puesto de trabajo de cada trabajador adscrito al seguro. La afiliación al Seguro de Cesantía es obligatoria para todos los ocupados cuyos contratos laborales se establecen desde el año 2003 y voluntaria para los contratos anteriores. No son cubiertos los empleados del sector público, los trabajadores por cuenta propia, los asalariados informales y las trabajadoras de

${ }^{6}$ La asignación de los estudiantes entre la educación técnico profesional y científico humanista se realiza en base al último grado cursado. En el caso de los estudiantes cuyo último grado de estudios es segundo medio, se procede a clasificarlos de acuerdo con la modalidad que prevalece en el establecimiento que cursan estudios.

${ }^{7}$ Estos alumnos no pueden distinguirse en la base de datos de aquellos que egresan de la educación media y no siguen estudios superiores, categoría que por lo tanto queda sujeta a algún grado de sobreestimación. 
Figura 1. DIAGRAMA PARA LA CONSTRUCCIÓN DE LA BASE DE DATOS

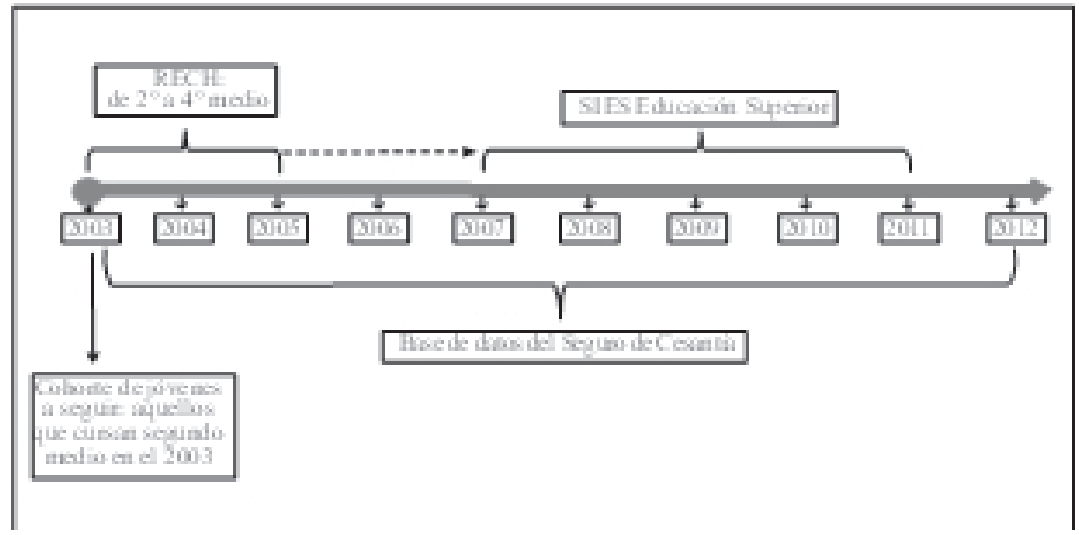

casa particular. No obstante, la elevada rotación laboral existente en Chile causa que la gran mayoría de los trabajadores quede registrada en la base del Seguro de Cesantía en aquellos períodos en que acceden a empleos que califican para cotización obligatoria. ${ }^{8}$

\section{TRAYECTORIAS EDUCACIONALES}

\section{1. ¿Quiénes estudian enseñanza media técnico profesional versus científico humanista?}

Investigaciones previas para Chile muestran que hay dos variables claves que intervienen en la distribución de los estudiantes de enseñanza media entre las modalidades de educación general y vocacional: el nivel socioeconómico y el rendimiento académico. ${ }^{9}$ La discusión que sigue tiene

${ }^{8}$ Así se desprende al comparar la tasa de ocupación de los egresados de la educación media técnico profesional de edad equivalente entre la encuesta Casen y los datos del Seguro de Cesantía, con resultados prácticamente idénticos.

${ }^{9}$ Farías (2013) discute los posibles determinantes de la elección entre enseñanza media TP versus $\mathrm{CH}$, teniendo por referencia las teorías de capital humano, capital cultural, capital social y elección de escuelas. De Iruarrizaga (2009) muestra que la elección de TP depende del nivel socioeconómico, del resultado en la prueba Simce y del ranking de notas del alumno en su establecimiento. Centro de Microdatos (2009) muestra que la distancia al establecimiento representa también una variable relevante en la elección de modalidad de estudios. 
carácter descriptivo, no siendo propósito de este documento la estimación de un modelo de elección de modalidades de enseñanza.

En este estudio la variable socioeconómica se mide en base a la escolaridad de la madre, según es informada en el cuestionario a los padres de la prueba Simce. Los alumnos se clasifican en cinco niveles según los estudios de sus madres: (i) educación básica incompleta o sin educación; (ii) educación básica completa; (iii) educación media incompleta; (iv) educación media completa; (v) educación superior, completa e incompleta. La distribución resultante entrega grupos de tamaños relativamente similares y por ello no difiere demasiado de una clasificación en quintiles. ${ }^{10}$

Por su parte, el rendimiento escolar se mide en base a los resultados del Simce de octavo básico. ${ }^{11}$ Ésta es una medida de logro escolar anterior al ingreso a la enseñanza media y, por tanto, no está influenciada por las características de la variable que es investigada. El análisis se realiza en la cohorte auxiliar, que cuenta con la medición del Simce de octavo básico. Si bien el resultado individual de la prueba Simce no es informado a los alumnos o sus padres, el puntaje Simce se correlaciona con indicadores observables del rendimiento académico, como el promedio de notas o con el ranking del estudiante en su curso, así como con la disposición general que los alumnos tienen respecto de los estudios. ${ }^{12}$

El cuadro 1 muestra el porcentaje de alumnos de la cohorte que sigue la modalidad técnico profesional, según nivel socioeconómico y quintil de puntaje de Simce de octavo básico. Resulta claro que la enseñanza media técnico profesional atrae preferentemente a estudiantes de familias vulnerables. Alrededor de tres de cada cinco estudiantes de los primeros niveles socioeconómicos siguen esta modalidad de estudios, mientras que en el segmento más acomodado la gran mayoría se adscribe a la enseñanza cien-

${ }^{10}$ Otros candidatos para realizar esta clasificación son la educación del padre y el ingreso familiar, sin embargo para muchos estudiantes no se dispone de información sobre la educación del padre mientras que el ingreso del hogar se reporta en tramos que son muy agregados para ser informativos.

${ }^{11}$ A lo largo del texto, el puntaje Simce considerado es el promedio de las pruebas de matemáticas y lenguaje.

12 Alrededor del 12\% de los estudiantes de la cohorte auxiliar no pueden ser clasificados por la variable socioeconómica. Ellos aparecen en el Registro de Estudiantes de Chile, pero no en la base de la prueba Simce. Ellos incluirían casos en que hay problemas con el identificador individual que permite unificar ambas bases, pero también a alumnos que no rinden el Simce por falta de motivación o porque sus establecimientos desincentivan que rindan la prueba. 
Cuadro 1. PORCENTAJE DE ALUMNOS EN MODALIDAD TÉCNICO PROFESIONAL SEGÚN NIVEL SOCIOECONÓMICO Y QUINTILES RESULTADOS SIMCE DE OCTAVO BÁSICO

\begin{tabular}{ccccccc}
\hline $\begin{array}{c}\text { Quintiles } \\
\text { Simce }\end{array}$ & \multicolumn{5}{c}{ Nivel socioeconómico } \\
\hline & 1 & 2 & 3 & 4 & 5 & Total \\
1 & 62,1 & 63,8 & 62,3 & 52,9 & 31,8 & 57,5 \\
2 & 64,4 & 63,4 & 59,2 & 48,0 & 24,1 & 53,0 \\
3 & 62,9 & 61,3 & 55,1 & 42,3 & 17,9 & 45,7 \\
4 & 60,2 & 57,3 & 49,2 & 34,0 & 11,0 & 34,8 \\
5 & 50,4 & 46,6 & 37,0 & 21,2 & 4,5 & 17,6 \\
Total & 61,8 & 60,5 & 54,6 & 38,6 & 12,2 & 41,3 \\
\hline
\end{tabular}

Fuente: RECh 2003 a 2011; Simce 2004 octavo básico, cohorte auxiliar.

tífico humanista. Asimismo, los alumnos que tienen puntajes más bajos en la prueba Simce de octavo básico optan preferentemente por la enseñanza media técnico profesional. Más de la mitad de aquéllos en los dos quintiles inferiores de resultados del Simce siguen la educación media técnico profesional, comparados con sólo un 18 por ciento en el quintil superior.

Es evidente que hay interrelación entre el nivel socioeconómico y el resultado del Simce. La correlación es positiva entre estas variables, de modo que los estudiantes de nivel socioeconómico alto tienden a tener buenos resultado en el Simce, mientras que los estudiantes de nivel socioeconómico bajo se concentran en la mitad inferior de los puntajes Simce (ver cuadro A.1 en Anexo). Éste es un hecho a tener presente cuando se interpretan los resultados del cuadro 1, que presenta porcentajes al interior de cada celda (pero no informa cuánta población hay en cada celda). ${ }^{13}$

Es interesante notar que los estudiantes en los niveles socioeconómicos inferiores tienden a optar por la enseñanza técnico profesional con relativa independencia del resultado obtenido en la prueba Simce. En cambio, en los estratos socioeconómicos medios y altos el resultado Simce cobra mayor relevancia, en tanto la probabilidad de seguir estudios técnico profesionales es mucho mayor en los estudiantes de bajo rendimiento académico.

\footnotetext{
${ }^{13}$ Igual advertencia aplica a los cuadros 5 y 6 , más adelante.
} 
En el Anexo se presentan los resultados de regresiones cuyos coeficientes proveen estimaciones más precisas del cambio en la probabilidad de estudiar TP frente a cambios en sus determinantes, controlando por el efecto de las demás variables. Los resultados muestran que la probabilidad de estudiar en la modalidad técnico profesional desciende en forma abrupta a partir del cuarto nivel socioeconómico y del cuarto quintil del resultado en el Simce, si bien el efecto de la variable socioeconómica es más importante en cuanto a su magnitud (cuadro A.2).

Los resultados descritos son consistentes con la premisa de que la enseñanza media técnico profesional constituiría una puerta de acceso más temprana al mercado laboral, por lo que atraería a quienes tienen una mayor necesidad de generar ingresos monetarios, así como a quienes tienen bajas expectativas de continuar estudios universitarios por presentar insuficiente rendimiento en los estudios generales.

\subsection{Expectativas de los padres sobre la escolaridad que alcanzarán sus hijos}

Las expectativas que tienen los padres sobre los logros educativos que alcanzarán sus hijos constituyen un factor relevante en la determinación de las trayectorias educacionales. Farías (2013) muestra que la decisión de elegir educación media técnico profesional es influenciada por las familias en la mayoría de los estudiantes considerados en su investigación para Chile.

Las expectativas se pueden medir en el cuestionario que responden los padres en la prueba Simce, que incluye la pregunta: "¿Qué nivel de estudios cree que completará el alumno?". En la medición realizada en 2004 (Simce de octavo básico, cohorte auxiliar), cerca del 80 por ciento de los padres esperaba que sus hijos obtendrían en el futuro un título de nivel superior (cuadro 2). El dato de mayor interés es la respuesta comparada según la modalidad educacional a la cual asistirán los hijos en la enseñanza media. La opción de estudios superiores es favorecida por el 87,4 por ciento de los padres cuyos hijos asistirán a la enseñanza científico humanista y el 66,9 por ciento de aquellos con hijos que asistirán a la enseñanza media técnico profesional. ${ }^{14}$ En el primer caso, la mayoría asumía que los estudios se realizarían en universidades; mientras que en el segundo caso la mayor

${ }^{14}$ Se hace notar que los datos utilizados son censales, por lo que no es necesario utilizar testeo de hipótesis para mostrar que las diferencias son significativas en la comparación de medias y otras estadísticas descriptivas. 
parte suponía que tendrían lugar en institutos profesionales o centros de formación técnica.

Este es un resultado importante, porque muestra que la mayor parte de los padres que matricula a sus hijos en enseñanza media técnico profesional no considera esta modalidad como etapa final de la educación de sus hijos, sino que proyecta la continuidad de los estudios hacia carreras técnicas o profesionales de nivel superior.

En la formación de expectativas intervienen factores subjetivos, como preferencias, aspiraciones y motivaciones, así como factores objetivos, como el desempeño escolar de los hijos, la calidad de la educación a la que pueden acceder, los recursos económicos del hogar, etcétera. El análisis de regresión muestra que el porcentaje de padres que espera continuidad de los estudios en el nivel superior crece con el nivel socioeconómico del hogar y el resultado del Simce en octavo básico; asimismo, es más elevado en el caso de las alumnas mujeres que los alumnos hombres (cuadro A.3 en Anexo).

\section{Cuadro 2. EXPECTATIVAS DE LOS PADRES EN OCTAVO BÁSICO SOBRE LA CONTINUIDAD DE ESTUDIOS DE LOS HIJOS, SEGÚN MODALIDAD DE ESTUDIO SEGUIDA POSTERIORMENTE EN ENSEÑANZA MEDIA (\%)}

\begin{tabular}{lccc}
\hline & TP & $\mathrm{CH}$ & Total \\
\hline \% que espera siga estudios superiores & 66,9 & 87,4 & 78,6 \\
• En universidades & 27,8 & 71,8 & 53,0 \\
- En instituto profesional o centro de & 39,1 & 15,6 & 25,6 \\
formación técnica & & & \\
\hline
\end{tabular}

Fuente: RECh 2003 a 2011; Simce octavo básico, 2004. Cohorte auxiliar.

\subsection{Distribución de estudiantes de enseñanza técnico profesional entre dependencias educacionales}

La enseñanza media técnico profesional se imparte en establecimientos municipales, particular subvencionados y de administración delegada a corporaciones privadas. Los dos primeros tipos congregan a la mayoría de los estudiantes, representando al 48 y 40,1 por ciento, respectivamente, de la cohorte técnico profesional en estudio. ${ }^{15}$

${ }^{15}$ La clasificación de la dependencia del establecimiento se realiza cuando el alumno cursa cuarto medio o cuando el último grado es efectivamente cursado en la enseñanza media. 
Los establecimientos municipales y particular subvencionados se financian con una subvención por alumno, cuyo monto varía dependiendo de la especialidad de estudios. En cambio, los liceos de administración delegada tienen un financiamiento basal del Estado y en su mayor parte son gestionados por organismos vinculados a gremios de sectores productivos, lo que en principio permitiría una mayor correspondencia entre los estudios realizados y las demandas de los mercados laborales.

La distribución socioeconómica de alumnos en la enseñanza media técnico profesional es muy similar entre las distintas dependencias educacionales (cuadro 3). Ello contrasta con la enseñanza media científico humanista, donde hay una marcada segmentación socioeconómica de los alumnos entre dependencias, puesto que los establecimientos pagados concentran al segmento más pudiente, la educación particular subvencionada al segmento medio-alto y la educación municipal a los alumnos vulnerables (cuadro A.4 en Anexo).

Sin embargo, hay diferencias en el nivel académico de los alumnos que ingresan a los diferentes tipos de establecimientos de la educación media técnico profesional (cuadro 4). Al interior de cada nivel socioeconómico se constata que los establecimientos delegados a corporaciones privadas captan alumnos con mejores resultados en la prueba Simce de octavo básico. La brecha a su favor es más elevada en los niveles socioeconómicos más bajos, del orden de 10 a 18 puntos, correspondiente del 20 al 36 por ciento de la desviación estándar de la variable.

Cuadro 3. DISTRIBUCIÓN DE LA COHORTE TÉCNICO PROFESIONAL POR NIVEL SOCIOECONÓMICO (\%)

\begin{tabular}{lcccccc}
\hline & \multicolumn{7}{c}{ Nivel socioeconómico } \\
\hline \multirow{3}{*}{ Municipal } & 1 & 2 & 3 & 4 & 5 & Total \\
Particular subvencionado & 34,4 & 22,3 & 20,1 & 19,6 & 3,7 & 100 \\
Corporación privada & 24,1 & 20,2 & 21,1 & 25,8 & 5,8 & 100 \\
Total & 24,1 & 20,4 & 22,8 & 27,6 & 5,1 & 100 \\
& 30,2 & 21,2 & 20,8 & 23,1 & 4,7 & 100 \\
\hline
\end{tabular}

Nota: Nivel socioeconómico se mide por años de escolaridad de la madre: 0-7; 8; 9-11; 12; 13 y más.

Fuente: RECh 2003 a 2011; Simce octavo básico 2004, cohorte auxiliar. 
Cuadro 4. PUNTAJE PROMEDIO EN SIMCE DE OCTAVO BÁSICO PARA ESTUDIANTES TÉCNICO PROFESIONALES, SEGÚN DEPENDENCIA Y NIVEL SOCIOECONÓMICO

\begin{tabular}{lcccccc}
\hline & \multicolumn{7}{c}{ Nivel socioeconómico } \\
\hline & 1 & 2 & 3 & 4 & 5 & Total \\
Municipal & 237 & 241 & 243 & 250 & 255 & 243 \\
Particular subvencionado & 238 & 244 & 246 & 255 & 261 & 249 \\
Corporación privada & 255 & 257 & 256 & 261 & 265 & 259 \\
Total & 239 & 244 & 246 & 254 & 260 & 247 \\
\hline
\end{tabular}

Fuente: RECh 2003 a 2011; Simce octavo básico 2004; cohorte auxiliar.

\subsection{Resultados en el Simce de segundo medio y en la Prueba de Selección Universitaria}

La prueba Simce mide el logro de los objetivos de aprendizaje de matemáticas, lenguaje y otras asignaturas cuyo currículo es compartido hasta segundo año de enseñanza media, por lo que no debieran haber diferencias promedio de resultados en el Simce de segundo medio entre alumnos con iguales características que sigan la modalidad científico humanista o técnico profesional.

Para evaluar este punto se necesita conformar grupos de alumnos comparables en los atributos que son relevantes en el logro de objetivos de aprendizaje. La disponibilidad de una medición de logro previo, como es el Simce de octavo básico, contribuye a resolver este problema, puesto que en el puntaje obtenido en esa prueba confluyen las características individuales y del entorno relevantes, como el nivel socioeconómico, el capital cultural, el lugar de residencia, la motivación por los estudios, las habilidades iniciales, etcétera. Por tanto, comparar los resultados del Simce de segundo medio entre estudiantes que obtuvieron un resultado similar en el Simce de octavo básico equivale a realizar la comparación entre alumnos que en octavo básico eran estadísticamente similares en las variables que inciden sobre los aprendizajes. ${ }^{16}$

El gráfico 1 presenta los resultados obtenidos de esta comparación de resultados. En el eje horizontal se ordena a los estudiantes en percentiles

${ }^{16}$ Por semejanza estadística debe entenderse que son comparables a nivel de promedios grupales, no individualmente. 
según el puntaje obtenido en el Simce de octavo básico, mientras que en el eje vertical se muestra la diferencia de puntajes en el Simce de segundo medio entre estudiantes que — al año siguiente - cursarán estudios científico humanistas versus técnico profesionales.

Los alumnos de la educación media técnico profesional obtienen en promedio 7 puntos menos en el Simce de segundo medio que sus pares en la educación científico humanista. Esta brecha no es constante, sino que se va ampliando a medida que mejora el rendimiento en el Simce de octavo básico. Para los alumnos en los primeros 40 percentiles la diferencia en el Simce de segundo medio es pequeña y menor a cinco puntos; para aquellos en los percentiles superiores se aproxima a los 20 puntos. Hay que tener presente que la desviación estándar del Simce de segundo medio es 45 puntos en los datos utilizados, de modo que la brecha promedio entre alumnos TP y CH representa un 15 por ciento de esta medida de dispersión.

La brecha de resultados descrita se originaría en diferencias en la enseñanza que reciben los alumnos de establecimientos de educación media de modalidad técnico profesional o científico humanista. Hay evidencia casuística y opiniones de actores que indicaría que algunos establecimientos introducen aspectos relacionados con la formación vocacional ya en primero y segundo medio, como fue su práctica histórica. Esto implica, por

Gráfico 1. DIFERENCIA EN PUNTAJE SIMCE SEGUNDO MEDIO ENTRE ALUMNOS CH YTP, SEGÚN PERCENTILES SIMCE OCTAVO BÁSICO

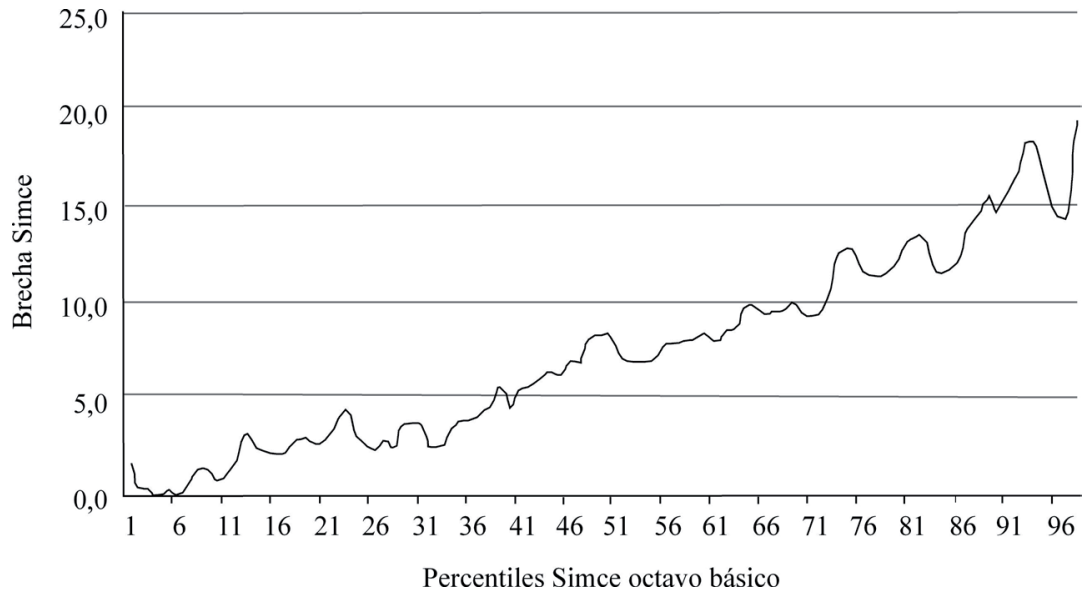

Fuente: Micro datos pruebas Simce segundo medio 2006 y octavo básico 2004. 
consideraciones de tiempo pedagógico, una menor atención a las asignaturas generales. ${ }^{17}$ También es posible que haya una diferencia motivacional por los estudios generales entre alumnos que ya han tomado la decisión de seguir una u otra modalidad de enseñanza media.

La ampliación de la brecha de los resultados Simce en los percentiles superiores de rendimiento académico previo puede obedecer a efectos sinérgicos en la acumulación de capital humano (Cunha et al. 2005). También puede ocurrir que haya inversiones adicionales de capital humano si se considera que los estudiantes en los percentiles superiores del Simce provienen de hogares con mayores recursos socioeconómicos. Para tal efecto, se realizó una regresión entre el resultado del Simce de segundo medio, el puntaje del Simce de octavo básico y el nivel socioeconómico, obteniéndose coeficientes positivos y significativos, pero que reducen sólo marginalmente el coeficiente del Simce de octavo básico (cuadro A.5 en Anexo). ${ }^{18}$ Ello muestra que la variable socioeconómica incide poco en la ampliación de la brecha de resultados descrita.

Para los alumnos que seguirán la enseñanza media técnico profesional los resultados de la regresión muestran que los alumnos en corporaciones privadas obtienen, en promedio, cerca de cinco puntos más en la prueba Simce que los alumnos en establecimientos particulares subvencionados, y cerca de ocho puntos más respecto de los alumnos en establecimientos municipales, después de controlar por diferencias en el nivel socioeconómico y rendimiento académico previo.

Un procedimiento análogo se aplica para los resultados de la Prueba de Selección Universitaria (PSU), que mide conocimientos de asignaturas de la enseñanza media hasta cuarto medio. Los alumnos de la modalidad técnico profesional tienen menos horas asignadas para el estudio de las materias medidas en la PSU y debieran llegar en inferioridad de condiciones para rendir la prueba. Una brecha negativa para los alumnos de enseñanza media técnico profesional es ahora un resultado esperado y la pregunta es sobre la magnitud del efecto.

${ }_{17}$ Un grupo minoritario de estudiantes asisten a establecimientos TP que imparten el ciclo completo de enseñanza básica y media. Para ellos podría suceder que las falencias en la educación general se inicien en la educación básica, "contaminando" nuestra línea base de octavo año. Sin embargo, es poco probable que esto suceda, porque la enseñanza básica opera con profesores que, por formación, son generalistas y porque este nivel de enseñanza siempre ha sido integrado.

${ }^{18}$ Específicamente, el coeficiente de interacción Simce 8B con la dummy de educación TP. 
Gráfico 2. DIFERENCIA EN PUNTAJE PSU ENTRE ALUMNOS CH YTP, SEGÚN PERCENTILES SIMCE OCTAVO BÁSICO

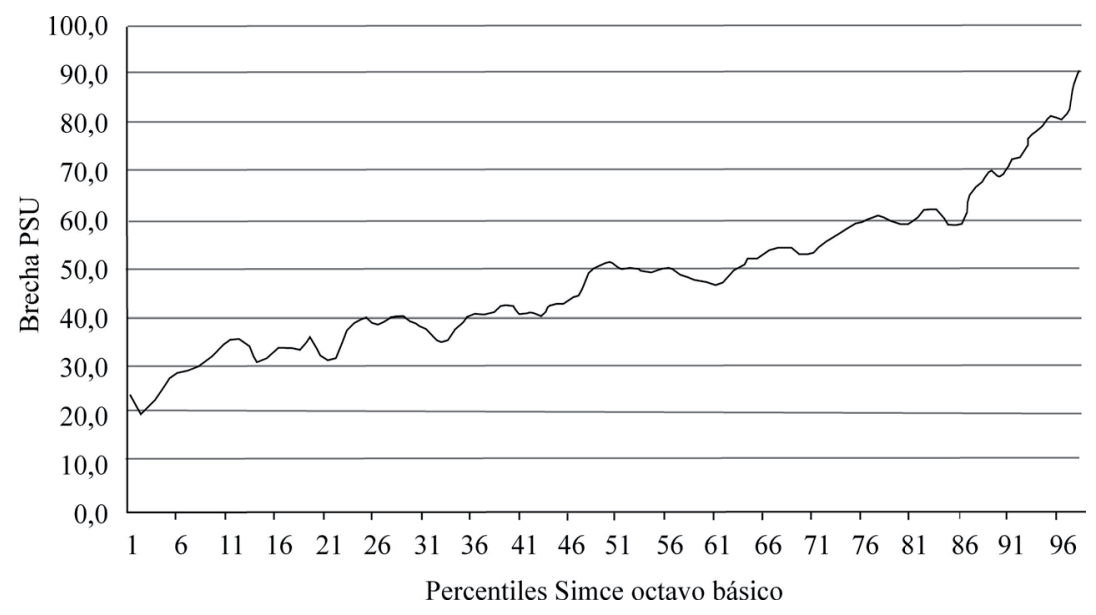

Fuente: Micro datos PSU 2006 (considera promedio lenguaje y matemáticas).

El gráfico 2 muestra que la brecha de resultados en la PSU es bastante más pronunciada que en el Simce, puesto que alcanza a un promedio de 47 puntos o 45 por ciento de la desviación estándar de la variable. ${ }^{19}$ La brecha de resultados aumenta cuando se compara a estudiantes con mayor puntaje en el Simce de octavo básico. La diferencia crece desde alrededor de 30 puntos en los percentiles inferiores del Simce hasta 80 o 90 puntos en los percentiles superiores. ${ }^{20}$

El análisis de regresión muestra que sólo una parte de la ventaja que acumulan los alumnos $\mathrm{CH}$ de mejor rendimiento inicial se debería a la variable socioeconómica (cuadro A.6 en Anexo). Al interior de la educación técnico profesional se tiene que los alumnos en corporaciones privadas obtienen en promedio cerca de 4 puntos más en la PSU que los alumnos en establecimientos particulares subvencionados, y cerca de 16 puntos más que los alumnos en establecimientos municipales.

Farías (2013) obtiene resultados relativamente similares y los vincula a la tesis de la distracción (diversion thesis), según la cual la educación

${ }^{19}$ Hay que notar que la PSU es rendida por un porcentaje mucho menor de egresados de la TP que CH (24\% versus $66 \%$ en la PSU evaluada) y serían aquellos que en principio tendrán la mejor preparación para rendir esta prueba.

${ }^{20} \mathrm{La}$ brecha es estadísticamente significativa en todos los percentiles. 
vocacional "captura" a alumnos vulnerables que hubiesen tenido un mayor potencial de desarrollo en la educación general, pero que optan por la modalidad técnico profesional por razones culturales o económicas, obteniendo en definitiva logros inferiores a los que podrían haber alcanzado en la educación general. Las investigaciones cualitativas de Ortiz (2009) y Sepúlveda et al. (2010) sugieren que la elección de la modalidad técnico profesional por parte de jóvenes de hogares vulnerables puede representar una estrategia racional bajo condiciones de incertidumbre, puesto que posibilita contar con un ingreso cierto al término de la enseñanza media y que puede ser destinado para financiar estudios superiores, entre otros fines.

Es importante hacer notar que no se cuenta con evaluaciones que midan las competencias específicas que se aprenden en la enseñanza media técnico profesional, lo que es una tarea pendiente del sistema educacional en Chile.

\subsection{Deserción en la enseñanza media}

La tasa de deserción es aquí definida como el porcentaje de alumnos de la cohorte que abandona la enseñanza media. Los datos muestran que los alumnos de la enseñanza media técnico profesional tienen tasas de deserción bastante más bajas que los alumnos de la enseñanza científico humanista, especialmente aquellos que pertenecen a hogares vulnerables o quienes tienen bajo rendimiento escolar en la enseñanza básica (cuadros 5 y 6). La tasa de deserción es alrededor de 2,5 veces más baja en la educación técnico profesional para estudiantes de los tres niveles socioeconómicos inferiores o de los dos quintiles más bajos del Simce de octavo básico. La diferencia es aún mayor para jóvenes que combinan ambas características, en cuyo caso la tasa de deserción es cercana a 10 por ciento en la enseñanza técnico profesional y alrededor del 30 por ciento en la modalidad científico humanista.

El análisis de regresión en el Anexo (cuadro A.7) muestra que la deserción de la enseñanza media responde más a diferencias en el rendimiento académico en la enseñanza básica que a diferencias en la condición socioeconómica. No obstante, ambos "factores de riesgo" importan más en la enseñanza científico humanista, en el sentido de que hay mayor pendiente de la tasa de deserción respecto de esas variables. También se constata que las mujeres desertan menos que los hombres en la enseñanza media. 
Cuadro 5. TASA DE DESERCIÓN DE ALUMNOS EN ENSEÑANZA MEDIA TÉCNICO PROFESIONAL SEGÚN NIVEL SOCIOECONÓMICO Y QUINTIL SIMCE DE OCTAVO BÁSICO (\%)

\begin{tabular}{crrrrrr}
\hline $\begin{array}{c}\text { Quintiles } \\
\text { Simce }\end{array}$ & \multicolumn{7}{c}{ Nivel socioeconómico } \\
\hline & 1 & 2 & 3 & 4 & 5 & Total \\
1 & 13,3 & 11,0 & 10,3 & 7,8 & 7,6 & 10,6 \\
2 & 7,4 & 7,2 & 6,6 & 4,8 & 5,4 & 6,4 \\
3 & 5,3 & 5,2 & 4,9 & 3,8 & 3,4 & 4,6 \\
4 & 4,1 & 3,8 & 3,5 & 2,9 & 2,4 & 3,3 \\
5 & 3,0 & 2,5 & 2,4 & 2,4 & 2,6 & 2,5 \\
Total & 8,3 & 7,0 & 6,4 & 4,5 & 4,2 & 6,2 \\
\hline
\end{tabular}

Fuente: RECh 2003-2011; Simce 2004, cohorte auxiliar.

Cuadro 6. TASA DE DESERCIÓN DE ALUMNOS EN ENSEÑANZA MEDIA CIENTÍFICO HUMANISTA SEGÚN NIVEL SOCIOECONÓMICO Y QUINTIL SIMCE DE OCTAVO BÁSICO (\%)

\begin{tabular}{ccccccc}
\hline $\begin{array}{c}\text { Quintiles } \\
\text { Simce }\end{array}$ & \multicolumn{7}{c}{ Nivel socioeconómico } \\
\hline & 1 & 2 & 3 & 4 & 5 & Total \\
1 & 32,9 & 31,5 & 28,6 & 20,8 & 15,4 & 26,1 \\
2 & 23,7 & 20,7 & 18,3 & 12,7 & 9,4 & 15,7 \\
3 & 16,7 & 13,2 & 12,0 & 7,3 & 5,0 & 8,9 \\
4 & 9,6 & 6,8 & 7,0 & 4,6 & 3,4 & 4,9 \\
5 & 3,4 & 3,5 & 2,7 & 2,0 & 1,8 & 2,0 \\
Total & 21,6 & 17,1 & 14,1 & 7,6 & 4,1 & 9,4 \\
\hline
\end{tabular}

Fuente: RECh 2003-2011; Simce 2004, cohorte auxiliar.

La deserción en la enseñanza media está asociada a la repetición de cursos y al atraso escolar. El 37 por ciento de los desertores había repetido una vez antes de segundo medio y el 18 por ciento había repetido al menos dos veces, según la información reportada en los cuestionarios a los padres del Simce de segundo medio. La repetición es un predictor de la deserción, porque informa sobre un mal desempeño educacional, pero también porque hace más probable que los alumnos cumplan con la edad requerida para 
ingresar a la educación de adultos cuando aún están cursando la enseñanza media regular.

La educación de adultos es una modalidad educativa para mayores de 18 años que permite hacer dos cursos en un año calendario en jornadas vespertinas. Ella congrega a un porcentaje no menor de los alumnos que abandonan la educación media regular. Para la cohorte principal se tiene que el 47 por ciento de los desertores de la educación media científico humanista y el 34 por ciento de los desertores de la enseñanza técnico profesional se matriculan en la educación de adultos en algún año del período 2004 a $2010 .^{21}$ No obstante, sólo una tercera parte de ellos logra egresar de la enseñanza media de adultos en el período bajo estudio.

\subsection{Acceso a la educación superior}

La tasa de acceso a la educación superior para la cohorte que cursa educación media técnico profesional es de 41,4 por ciento. Éste es un porcentaje muy significativo y señala que esta modalidad de estudios no puede seguir considerándose como una fase terminal de enseñanza. ${ }^{22} \mathrm{La}$ mayor parte de este grupo asiste a centros de formación técnica e institutos profesionales (61,9 por ciento). En cambio, los jóvenes que estudiaron en la modalidad científico humanista tienen una tasa de acceso a la educación superior del 67,1 por ciento y cursan preferentemente sus estudios en universidades ( 70 por ciento). ${ }^{23}$

${ }^{21}$ El porcentaje es más elevado entre los desertores de los niveles socioeconómicos acomodados. Así, en la modalidad científico humanista se matricula en la educación de adultos el 44 por ciento de los desertores del nivel socioeconómico más bajo y el 60 por ciento del nivel más alto. En la enseñanza técnico profesional los respectivos porcentajes son 31 y 50 .

${ }^{22}$ Este dato refiere a la cohorte en estudio, cuyo ingreso a la educación superior parte en 2006. El porcentaje de estudiantes de enseñanza media que accede a la educación superior ha crecido en el tiempo: en el bienio 2011-2012 el número de matriculados en el primer año es 47 por ciento mayor respecto del bienio 20062007 (para un total de estudiantes de enseñanza media más o menos estable en el tiempo).

${ }^{23}$ A nivel del conjunto de la cohorte, el 55,6 por ciento ingresa a la educación superior. Entre ellos, el 60,1 por ciento cursa estudios en universidades, 25,1 por ciento en institutos profesionales y 14,8 por ciento en centros de formación técnica. Estas cifras no son comparables con las estadísticas que se publican sobre matrícula en educación superior, puesto que se trata del dato acumulado para el conjunto de la cohorte, mientras que las estadísticas habituales están referidas a la matrícula en un determinado año calendario. 
El ingreso a la educación superior es diferido en el tiempo. Para los egresados de la cohorte científico humanista que ingresan a la educación superior, cerca de la mitad lo hace en 2006, la tercera parte en 2007 y el otro 15 por ciento en los años siguientes. Para los estudiantes de la enseñanza media técnico profesional, el rezago es mucho mayor, puesto que sólo cerca del 20 por ciento ingresa en 2006, 35 por ciento en 2007 y el 45 por ciento entre 2008 y 2011.

El ingreso diferido de los egresados de la educación técnico profesional puede deberse a dos razones: la primera es específica al período analizado y reside en la disponibilidad de ayuda financiera para pagar por los estudios superiores. En estos años (2006 a 2011), se produce un fuerte aumento de créditos y becas para financiar estudios en centros de formación técnica e institutos profesionales, donde estudia la mayoría de los egresados de la educación técnico profesional. Es por ello plausible que a medida que se hacía disponible el financiamiento se integraban más miembros de la cohorte a la educación superior. ${ }^{24} \mathrm{La}$ segunda razón es que el acceso diferido sea consecuencia de la inserción laboral temprana por parte de los jóvenes egresados de la educación media técnico profesional. ${ }^{25}$

Cuadro 7. INSTITUCIONES DONDE SE REALIZAN LOS ESTUDIOS SUPERIORES (\%)

\begin{tabular}{lccc}
\hline & TP & $\mathrm{CH}$ & Todos \\
\hline Centro de formación técnica & 23,4 & 10,9 & 14,8 \\
Instituto profesional & 38,5 & 19,0 & 25,1 \\
Universidad & 38,1 & 70,1 & 60,1 \\
Total & 100 & 100 & 100 \\
\hline
\end{tabular}

Fuente: RECh 2003-2011; SIES 2007-2011.

Para examinar el punto se realizó un análisis comparado con la cohorte que egresó de la educación media en el año 2007. Esta cohorte presenta un patrón de ingreso a la educación superior tanto o más diferido que la cohorte que egresa en 2005, a pesar de que tiene mayor acceso a becas y

${ }^{24}$ La colocación de financiamiento estudiantil en la forma de Créditos con Aval del Estado y Becas Nuevo Milenio aumentó desde MM\$ 61,2 en 2006 a MM\$ 294,8 en 2011.

${ }^{25}$ En la sección 3 se presenta en detalle la inserción laboral de la cohorte. 
créditos para financiar el pago por la educación superior. Ello apunta a la inserción laboral temprana como causal más probable del ingreso diferido a la educación superior. ${ }^{26}$

Interesa también caracterizar el acceso a la educación superior en términos de la calidad de las instituciones en que se cursan los estudios. Esta variable puede ser aproximada por los años en que la institución ha sido acreditada, que fluctúan entre 0 y 7 . Un mayor número de años de acreditación señalaría que la institución mantiene estándares de calidad más elevados. ${ }^{27}$

La mayoría de los alumnos que provienen de la enseñanza media técnico profesional estudia en universidades acreditadas menos años, comparados con los egresados de la enseñanza científico humanista. Así, el 51 por ciento de la cohorte asiste a universidades no acreditadas o con dos a tres años de acreditación, comparado con el 26,9 por ciento de los estudiantes que provienen de la enseñanza media $\mathrm{CH}$ (cuadro 8). A nivel de los institutos profesionales, sigue existiendo una brecha de calidad entre las entidades a las cuales asisten los alumnos provenientes de las diferentes modalidades de enseñanza media, pero es mucho menos pronunciada; a nivel de los centros de formación técnica no se observan diferenciales de calidad entre los estudiantes TP y $\mathrm{CH}$.

En suma, los estudiantes de la cohorte de educación media técnico profesional ingresan en número suficiente a la educación superior para que esa modalidad de estudios medios deje de ser considerada una etapa

${ }^{26}$ La cohorte técnico profesional que egresa en 2007 presenta la siguiente distribución de acceso a la educación superior: 23,5 por ciento en el primer año, 42 en el segundo, 21 en el tercero y 13,5 por ciento en el cuarto. Los porcentajes se calculan sobre el total que ingresa a la educación superior en los cuatro años siguientes al egreso de la educación media. Las cifras correspondientes a la cohorte que egresa en 2005 son, respectivamente, 34, 35, 19,4 y 11,6 por ciento (considerando sólo a los que ingresan los cuatro primeros años).

${ }^{27}$ La medición de la calidad de las universidades es una materia compleja por la diversidad de funciones y de misiones institucionales. La acreditación institucional existente en Chile es realizada por pares académicos y se basa en evaluar el cumplimiento del proyecto de la institución y el funcionamiento de mecanismos eficaces de autorregulación y de aseguramiento de la calidad. Una medición alternativa clasifica a las universidades mediante indicadores objetivos, que se basan en atributos de los profesores, estudiantes, proceso formativo y gestión institucional (Grupo de Estudios Avanzados Universitas), pero el orden resultante no difiere en lo sustancial de la acreditación institucional. Un desafío pendiente en ambos casos es evaluar los aprendizajes efectivos de los estudiantes y su empleabilidad. 
terminal de enseñanza. La distribución de alumnos entre instituciones de educación superior según modalidades de enseñanza media es consistente con la naturaleza de los estudios respectivos (académicos versus técnico profesionales); con las expectativas que tenían los padres respecto a los estudios de los hijos cuando ellos cursaban octavo año básico, y con los puntajes que obtienen ambos grupos de estudiantes en la Prueba de Selección Universitaria.

Cuadro 8. DISTRIBUCIÓN DE LA MATRÍCULA SEGÚN AÑOS DE ACREDITACIÓN DE LAS INSTITUCIONES DE ESTUDIOS

\begin{tabular}{crrrrrr}
\hline $\begin{array}{c}\text { Años de } \\
\text { acreditación }\end{array}$ & \multicolumn{2}{c}{ Universidades } & \multicolumn{2}{c}{ Institutos profesionales } & \multicolumn{2}{c}{$\begin{array}{c}\text { Centros de formación } \\
\text { técnica }\end{array}$} \\
\cline { 2 - 7 } & TP & \multicolumn{1}{c}{ CH } & \multicolumn{1}{c}{ TP } & CH & TP & CH \\
\hline Sin acreditar & 13,4 & 6,8 & 7,4 & 5,6 & 29,2 & 24,1 \\
$2-3$ & 37,6 & 20,1 & 5,5 & 5,8 & 6,0 & 4,6 \\
$4-5$ & 41,5 & 50,9 & 52,8 & 40,9 & 28,6 & 35,0 \\
$6-7$ & 7,5 & 22,1 & 34,3 & 47,8 & 36,2 & 36,3 \\
Total & 100,0 & 100,0 & 100,0 & 100,0 & 100,0 & 100,0 \\
\hline
\end{tabular}

Fuente: RECh 2003-2011; SIES 2007-2011; Consejo Nacional de Educación.

\subsection{Deserción en los estudios superiores}

En este estudio se entiende por deserción de los estudios superiores el abandono del sistema, antes que el simple cambio entre carreras o instituciones. Se hace notar que las trayectorias en educación superior no son aún definitivas y están sujetas a cambios futuros: quienes abandonan pueden retornar a los estudios; una parte de aquellos que están estudiando desertaran, etcétera.

A tal respecto, sorprende el elevado porcentaje de estudiantes de la cohorte que provienen de la enseñanza media técnico profesional y que abandona sus estudios superiores: el 32,6 comparado con el 19 entre los egresados de la enseñanza media científico humanista. Este resultado revierte el obtenido a nivel de la enseñanza media, donde los alumnos de educación técnico profesional tienen la menor tasa de deserción. ${ }^{28}$

${ }^{28}$ Farías (2013) obtiene un resultado similar en base a la variable persistencia de los estudios superiores. 
La deserción en la educación superior es especialmente elevada para los estudiantes hombres provenientes de establecimientos técnico profesionales. El 35,5 por ciento de quienes ingresaron a la educación superior no termina sus estudios, comparado con el 20,4 por ciento de los hombres egresados de la enseñanza científico humanista. Para las estudiantes mujeres, las tasas respectivas son 29,4 y 17,9 por ciento.

El análisis de regresión muestra que la mayor tasa de deserción que presentan los egresados de la educación técnico profesional respecto de los egresados de la formación científico humanista se reduce a menos de la mitad cuando se controla por el nivel socioeconómico y el tipo de instituciones donde se realizan los estudios superiores. Se sigue que la mayor deserción que presentan estos jóvenes, en buena parte, sería atribuible a su vulnerabilidad socioeconómica y al hecho de estudiar en institutos profesionales y centros de formación técnica, atributos que en sí mismos tienen asociada una mayor probabilidad de deserción de los estudios. ${ }^{29}$

Al interior de la cohorte técnico profesional se constata que la tasa de deserción es más baja para los jóvenes que trabajan y estudian, lo cual desvirtúa una de las posibles explicaciones para la mayor tasa de deserción del grupo (que la deserción sea causada por la dificultad de conciliar ambas actividades). Asimismo, se constata que la tasa de deserción es más elevada en las instituciones de educación superior de menor calidad (medida por los años de acreditación).

\section{INSERCIÓN LABORAL}

El análisis de la inserción laboral se organiza en base a los niveles de escolaridad alcanzados al año 2011. Para ello se clasifica a la cohorte en los siguientes grupos: titulados en las instituciones de educación superior, estudiantes en educación superior, desertores de la educación superior, egresados de la enseñanza media que no continúan estudios superiores (en adelante, egresados de enseñanza media) y desertores de la enseñanza media. Hay que notar que se trata de categorías que distan de ser homogéneas

${ }^{29}$ Ver cuadro A.8 en Anexo. La regresión considera a los estudiantes con información sobre la institución donde se realizan estudios superiores, variable que se registra a partir de 2008 en el SIES. La regresión no considera por tanto a quienes desertaron en los años 2006 y 2007. Las estadísticas de deserción citadas en el texto más arriba sí incluyen esos casos. 
en cuanto a su tamaño y que presentan una muy distinta distribución entre los jóvenes provenientes de la educación técnico profesional y científico humanista, un dato clave a la hora de interpretar los resultados de esta sección (cuadro 9).

Cuadro 9. DISTRIBUCIÓN DE LA COHORTE SEGÚN NIVELES EDUCACIONALES EN $2011(\%)$

\begin{tabular}{lcc}
\hline & \multicolumn{2}{c}{ Modalidad de estudios secundarios } \\
& TP & CH \\
\hline Titulados universidades & 2,6 & 9,4 \\
Titulados institutos profesionales & 3,1 & 3,7 \\
Titulados centros de formación técnica & 2,5 & 2,9 \\
Estudiantes de educación superior & 19,3 & 38,1 \\
Abandonaron los estudios superiores & 13,7 & 13,5 \\
Egresados educación media & 43,7 & 15,1 \\
Desertores de la educación media & 15,1 & 17,3 \\
Total & 100,0 & 100,0 \\
\hline
\end{tabular}

Fuente: RECh 2003-2011; SIES 2007-2011.

\subsection{Participación laboral}

Los jóvenes de la cohorte técnico profesional presentaban en el año 2011 una significativa mayor presencia en el mercado laboral que los provenientes de la enseñanza científico humanista, tanto a nivel de la tasa de ocupación como del número acumulado de meses trabajados a esa fecha (cuadro 10). Así, el 69,3 por ciento de ellos registra ocupación en el año 2011 y ha acumulado un promedio de 46 meses trabajados. Para la cohorte que cursó estudios científico humanistas, las cifras respectivas son 50,8 por ciento y 32 meses. La tasa de ocupación es aquí definida como el porcentaje de miembros de la cohorte que trabajaron con remuneración en el año 2011.

Así, los jóvenes de la educación técnico profesional presentaban una tasa de ocupación que es 18,5 puntos porcentuales más elevada y habían acumulado 14 meses más de experiencia laboral. La brecha se reduce a 11,3 puntos en la tasa de ocupación y 11,5 meses acumulados de trabajo si 
se excluye a quienes están estudiando en el nivel superior, que es la categoría donde los jóvenes provenientes de la enseñanza científico humanista presentan la menor tasa de participación laboral y que introduce un sesgo en su contra en la cifra agregada.

La mayor participación laboral de los jóvenes que estudiaron en la enseñanza técnico profesional rige para todas las categorías educacionales, sean titulados de la educación superior, egresados de la enseñanza media, desertores de la educación media y demás grupos. Para los titulados de educación superior se tiene que la mayor parte de quienes provienen de la educación técnico profesional ya trabajaba cuando cursaba sus estudios superiores, mientras que quienes provienen de la enseñanza científico humanista estarían en su mayor parte recién incorporándose al mercado laboral. Para los egresados de la enseñanza media y demás grupos se tiene que la mayor presencia laboral reflejaría ventajas en la formación de competencias laborales en la enseñanza media técnico profesional y una probable mayor predisposición hacia el trabajo remunerado por parte de este grupo.

Cuadro 10. TASA DE OCUPACIÓN EN 2011 Y MESES ACUMULADOS DE TRABAJO DE LA COHORTE

\begin{tabular}{|c|c|c|c|c|}
\hline & \multicolumn{2}{|c|}{$\begin{array}{l}\text { \% ocupados sobre total } \\
\text { del grupo en la cohorte }\end{array}$} & \multicolumn{2}{|c|}{$\begin{array}{l}\text { Promedio meses con } \\
\text { ingresos } 2003 \text { a } 2011^{*}\end{array}$} \\
\hline & TP & $\mathrm{CH}$ & TP & $\mathrm{CH}$ \\
\hline Titulados universidades & 71,4 & 60,3 & 31,9 & 19,3 \\
\hline Titulados institutos profesionales & 79,6 & 70,9 & 40,2 & 28,1 \\
\hline $\begin{array}{l}\text { Titulados centros de formación } \\
\text { técnica }\end{array}$ & 72,4 & 63,3 & 38,8 & 31,2 \\
\hline $\begin{array}{l}\text { Estudiantes de educación } \\
\text { superior }\end{array}$ & 62,6 & 36,7 & 41,4 & 22,0 \\
\hline $\begin{array}{l}\text { Abandonaron los estudios } \\
\text { superiores }\end{array}$ & 77,4 & 59,9 & 46,0 & 32,7 \\
\hline Egresados educación media & 71,5 & 57,1 & 50,1 & 44,2 \\
\hline Desertores de la educación media & 61,4 & 58,1 & 44,5 & 43,4 \\
\hline Total & 69,3 & 50,8 & 46,0 & 32,0 \\
\hline $\begin{array}{l}\text { Total sin estudiantes educación } \\
\text { superior }\end{array}$ & 70,9 & 59,6 & 47,0 & 35,8 \\
\hline
\end{tabular}

*: para quienes están trabajando en el 2011.

Fuente: RECh 2003-2011; SIES 2007-2011; Seguro de Cesantía 2003-2012. 


\subsection{Remuneraciones}

El perfil de remuneraciones que muestran los jóvenes de la cohorte sigue un patrón esperado respecto de la escolaridad, en tanto más años de educación tienen asociada una mayor remuneración promedio (cuadro 11). Sin embargo, el diferencial de remuneraciones es relativamente acotado, puesto que corresponde a la etapa inicial del ciclo de vida laboral y tenderá a ampliarse a medida que se acumule capital humano específico en los trabajos. ${ }^{30}$

¿Cómo se comparan las remuneraciones obtenidas por los jóvenes que estudiaron enseñanza media técnico profesional y científico humanista? Los datos del Seguro de Cesantía muestran que para el año 2012 la remuneración promedio de los jóvenes provenientes de la enseñanza técnico profesional tiende a ser más alta en la mayor parte de las categorías educacionales. ${ }^{31}$

Cuadro 11. REMUNERACIONES EN EL AÑO 2012 (MILES \$, BRUTOS)

\begin{tabular}{|c|c|c|c|c|}
\hline & \multicolumn{2}{|c|}{$\begin{array}{l}\text { Remuneración } \\
\text { promedio }\end{array}$} & \multicolumn{2}{|c|}{ Brecha TP vs CH (\%) } \\
\hline & TP & $\mathrm{CH}$ & Sin ajustar & Ajustada $\#$ \\
\hline Titulados universidades & 551,5 & 624,0 & $-11,6^{* \star *}$ & $-9,9^{* \star *}$ \\
\hline Titulados institutos profesionales & 508,3 & 468,4 & $8,5^{\star \star *}$ & 1,8 \\
\hline $\begin{array}{l}\text { Titulados centros de formación } \\
\text { técnica }\end{array}$ & 439,5 & 381,3 & $15,3^{* * *}$ & $5,0^{*}$ \\
\hline $\begin{array}{l}\text { Abandonaron los estudios } \\
\text { superiores }\end{array}$ & 389,9 & 353,9 & $10,2^{* \star \star}$ & $-0,2$ \\
\hline Egresados educación media & 349,8 & 328,1 & $6,6^{* * *}$ & 1,0 \\
\hline Desertores de la educación media & 298,3 & 299,2 & $-0,3$ & $-2,3^{*}$ \\
\hline
\end{tabular}

\# Nota: La brecha ajustada corresponde a la diferencia porcentual de remuneraciones controlando por la experiencia laboral, sexo y nivel socioeconómico del hogar paterno. Los asteriscos señalan significancia estadistica al $1 \%\left({ }^{* *}\right), 5 \%\left({ }^{* *}\right)$ y $10 \%\left(^{*}\right)$.

Fuente: RECh 2003-2011; SIES 2007-2011; Seguro de Cesantía 2003-2012.

${ }^{30}$ En la cohorte de estudio hay una diferencia de 2:1 entre las remuneraciones promedio de los titulados universitarios y los desertores de la enseñanza media; en la cohorte de 30 a 40 años esta diferencia aumenta a 4:1 (Casen 2011).

${ }^{31}$ Los salarios que se reportan corresponden al promedio mensual entre enero y octubre de 2012, último período para el cual se dispone de información (el dato promedio incluye sólo los meses con remuneración). 
Así, para los ya titulados en institutos profesionales hay una diferencia positiva promedio de 8,5 por ciento a favor de la cohorte de enseñanza media técnico profesional; para los titulados de centros de formación técnica la brecha es 15,3 por ciento; para quienes abandonan la educación superior es 10,2 por ciento, y para los egresados de la enseñanza media sin estudios posteriores la brecha promedio asciende a 6,6 por ciento. En cambio, entre los titulados universitarios hay una diferencia de ingresos de 11,6 por ciento a favor de los egresados de la educación media científico humanista.

Hay dos resguardos importantes a tener en cuenta sobre estos resultados. Primero, los ocupados que provienen de la educación técnico profesional tienen acumulada una mayor experiencia laboral y este hecho explica parte de la mayor remuneración que obtienen en el mercado laboral. Cuando se ajustan los resultados por el efecto de las diferencias en experiencia laboral, género y nivel socioeconómico, se tiene que la brecha de remuneraciones a favor de estos jóvenes mantiene significancia estadística sólo en el caso de los titulados de centros de formación técnica (cuadro 11, última columna). Segundo, las comparaciones de ingreso descritas no representan efectos causales de la educación sobre los salarios, porque no se dispone de controles sobre la distribución de habilidades que tenían los jóvenes previo a su ingreso a la educación media ni del clima cultural en sus hogares que afecta las motivaciones y expectativas de los estudiantes. Asimismo, hay importantes diferencias en la tasa de participación laboral según modalidad de estudios en la educación media, que muy probablemente afecten la comparación de remuneraciones entre ambos grupos.

A estos efectos hay que tener en cuenta los resultados obtenidos por Bucarey \& Urzúa (2013a), quienes reportan una reducción del retorno de la educación media técnico profesional cuando se controla por atributos no observables de los jóvenes mediante el uso de variables instrumentales. Esto es, parte del retorno económico que se observa entre los ocupados se debería a características personales antes que a la enseñanza vocacional propiamente tal.

Otra comparación de interés es al interior de la cohorte técnico profesional, entre egresados de la enseñanza media y titulados de la educación superior. En promedio, un joven que se titula en un instituto profesional obtiene una remuneración inicial que es 45 por ciento superior a la que percibe un egresado de la enseñanza media. En el caso de un titulado de un centro de formación técnica, la brecha respecto de un egresado de la ense- 
ñanza media es de 25 por ciento. Un cálculo simple muestra que los citados diferenciales cubren el valor de los aranceles de los respectivos estudios superiores en un período inferior a tres años. ${ }^{32}$

\section{3. Áreas de estudio en la enseñanza media técnico profesional e inserción laboral de los egresados}

La relevancia laboral que tienen las áreas de estudio de la enseñanza media técnico profesional puede ser analizada a través de los egresados del nivel que no prosiguen estudios superiores, porque sus competencias laborales están directamente relacionadas con las adquiridas en los estudios medios. El grupo es también de interés por su carácter masivo, pues representa al 44 por ciento de la cohorte técnico profesional o 20 por ciento de toda la cohorte, y porque su nivel de estudios corresponde a la misión objetivo que originalmente se planteaba la enseñanza media técnico profesional; es decir, preparar a los jóvenes para que se incorporen al mercado del trabajo a su egreso de la enseñanza media.

Hasta mediados del año 2013, la enseñanza técnica profesional contenía catorce sectores de estudios, que se desagregan en 46 especialidades. La clasificación del grupo en términos de los sectores entrega subgrupos de tamaño muy disímiles. Por ello se desagregaron los sectores más numerosos en términos de sus principales especialidades, dando lugar a un total de 21 sectores/especialidades de estudio, que en adelante se denominan "áreas de estudios". 33

El perfil de remuneraciones del grupo se muestra en el cuadro 12, en el cual las áreas de estudios se ordenan de acuerdo con la remuneración promedio obtenida por los jóvenes de la cohorte en el año 2012. Por regla general, las áreas mejor remuneradas se vinculan a oficios industriales, como mecánica industrial, electricidad, química, construcciones metálicas y mecánica automotriz. En cambio, las remuneraciones más bajas corresponden mayoritariamente a la producción de servicios, como administración, hotelería, secretariado, alimentación y programas sociales.

${ }^{32}$ La remuneración es 12 por ciento mayor en promedio cuando los estudios en centros de formación técnica o institutos profesionales se realizan en especialidades similares a las cursadas en la enseñanza media técnico profesional (sin ajustar por otras variables). Este es un dato sólo referencial, puesto que la correspondencia de los sectores de estudios en los niveles medio y superior no es exacta en las bases de datos utilizadas (RECh, SIES y Seguro de Cesantía).

${ }^{33}$ Ver Anexo, cuadro A.9. 
La brecha salarial entre estas ocupaciones ha aumentado en el tiempo. Al primer año después del egreso de la enseñanza media había una diferencia de 21 por ciento de remuneración promedio entre los oficios industriales y de servicios; al sexto año después del egreso la brecha había crecido a 42 por ciento. Ello podría entenderse como reflejo del mayor aprendizaje en los puestos de trabajo en los oficios industriales, donde la acumulación de capital humano genera mayores remuneraciones, aunque también hay que considerar que la demanda por algunos de estos oficios experimentó un mayor crecimiento en este período (2006 a 2012), por efecto del aumento de precios de los productos de exportación.

Las diferencias de remuneraciones entre áreas de estudios responden, por una parte, a factores de demanda, que otorgan mayor valor al trabajo realizado en los sectores productivos que experimentan más demanda de mercado. También hay atributos de los oferentes de trabajo, que influyen en las remuneraciones y que difieren entre las áreas de estudio, como es el género, la calidad de la educación recibida, experiencia laboral acumulada y nivel socioeconómico del hogar paterno.

El análisis de regresión muestra que las diferencias salariales promedio entre áreas de estudios explican alrededor del 10 por ciento de la varianza del salario del grupo y que la adición de los atributos individuales permiten dar cuenta del 23 por ciento de la citada varianza (cuadro A.10 en Anexo). En promedio, las mujeres ganan alrededor de \$ 57.000 menos que los hombres; un mes adicional de experiencia laboral se asocia a un aumento cercano a $\$ 3.500$ en la remuneración promedio; haber cursado los estudios de enseñanza media en un establecimiento del quintil superior de calidad tiene asociado un premio de ingresos de alrededor de $\$ 46.000$ respecto del quintil inferior, ${ }^{34} \mathrm{y}$ hay una diferencia de $\$ 26.000$ en la remuneración promedio entre personas del quintil superior e inferior de la variable socioeconómica. Estas brechas de ingresos ya consideran el efecto de los demás variables de la regresión. Los efectos no condicionales, o sin ajustar por las demás variables, son mucho más elevados.

${ }^{34}$ No se cuenta con medidas directas de la calidad de los establecimientos técnico profesionales. En la regresión se aproxima esta variable a través del porcentaje de alumnos desertores a nivel del establecimiento. La hipótesis subyacente es que el incentivo para desertar depende de la calidad de la educación que se recibe, por lo que la primera variable sería informativa de los niveles de la segunda. No obstante, puede haber otras interpretaciones para la relación entre calidad y deserción a nivel de establecimientos. 
Cuadro 12. SALARIO Y TASA DE OCUPACIÓN PROMEDIO POR ÁREA DE ESTUDIOS, ENTRE LOS EGRESADOS DE LA EDUCACIÓN MEDIA TÉCNICO PROFESIONAL QUE NO SIGUEN ESTUDIOS SUPERIORES (áreas ordenados por remuneración promedio bruta)

\begin{tabular}{|c|c|c|c|c|}
\hline Área de estudios & $\begin{array}{c}\text { Remuneración } \\
\text { promedio } \\
2012, \\
\text { miles de } \$\end{array}$ & $\begin{array}{c}\text { Remuneración } \\
\text { mediana } 2012 \\
\text { miles de } \$\end{array}$ & $\begin{array}{c}\text { Tasa } \\
\text { ocupa- } \\
\text { ción } 2011\end{array}$ & $\begin{array}{l}\% \text { de } \\
\text { total }\end{array}$ \\
\hline Minero & 531,6 & 435,8 & 86,8 & 0,9 \\
\hline Mecánica industrial & 433,1 & 358,0 & 82,4 & 4,6 \\
\hline Electricidad & 395,5 & 349,3 & 82,1 & 5,9 \\
\hline Químico & 383,8 & 326,5 & 72,3 & 0,8 \\
\hline Construcciones metálicas & 378,2 & 314,3 & 83,1 & 3,8 \\
\hline Mecánica automotriz & 372,1 & 314,5 & 80,2 & 7,5 \\
\hline Electrónica & 366,3 & 322,6 & 80,3 & 4,8 \\
\hline Gráfica & 356,8 & 304,9 & 71,8 & 1,1 \\
\hline Maritimo & 346,2 & 298,4 & 79,0 & 1,9 \\
\hline Construcción & 343,4 & 302,8 & 78,4 & 6,5 \\
\hline Telecomunicaciones & 340,1 & 300,6 & 76,0 & 1,5 \\
\hline Contabilidad & 306,6 & 274,0 & 72,6 & 7,6 \\
\hline Maderero & 306,3 & 280,1 & 74,5 & 2,7 \\
\hline Agropecuario & 302,2 & 266,1 & 73,3 & 5,4 \\
\hline Ventas & 301,5 & 271,8 & 70,6 & 4,1 \\
\hline Administración & 300,3 & 265,8 & 68,3 & 13,9 \\
\hline Hoteleria y turismo & 284,4 & 257,1 & 68,3 & 2,4 \\
\hline Secretariado & 276,6 & 251,0 & 63,7 & 5,5 \\
\hline Alimentación & 271,0 & 251,4 & 67,8 & 10,0 \\
\hline Programas y proyectos sociales & 251,1 & 240,3 & 63,9 & 7,6 \\
\hline Confección & 236,7 & 228,8 & 52,5 & 1,7 \\
\hline Total & 349,8 & 297,4 & 72,1 & 100,0 \\
\hline
\end{tabular}

Fuente: RECh 2003-2011; SIES 2007-2011; Seguro de Cesantía 2003-2012. Las remuneraciones son brutas. 
La brecha de ingreso entre las áreas mejor y peor pagadas, excluyendo a la minería, disminuyen desde \$239.800 a \$ 131.600 una vez que se consideran las diferencias que hay entre los ocupados en cuanto a género, experiencia laboral, calidad de los establecimientos y nivel socioeconómico. No obstante, el orden de áreas de estudio según nivel de ingreso laboral obtenido por los egresados se modifica sólo marginalmente después de tomar en cuenta los diferentes atributos de los ocupados. Ello sugiere que los oferentes de trabajo con atributos más valorados en el mercado del trabajo tienden a especializarse en las áreas que pagan mejor, ampliando las diferencias de ingresos que se observan entre ellas.

Es interesante hacer notar que en el trabajo de Bucarey \& Urzúa (2013a) se muestra que el orden relativo de remuneraciones de egresados de la educación media técnico profesional no se altera cuando se controla por sesgo de selección, si bien estos autores trabajan a nivel más agregado (cinco ramas de la educación TP).

\subsection{Diferencias salariales de género}

Es un hecho conocido del mercado laboral que las mujeres obtienen remuneraciones más bajas, a igualdad de su capital humano y otros determinantes de la productividad laboral. La pregunta que aquí interesa responder es si la educación media técnico profesional acentúa o reduce estas brechas salariales.

El cuadro 13 muestra que las mujeres de la cohorte obtienen remuneraciones inferiores para cada nivel educacional y que la brecha de salarios es más acentuada entre quienes provienen de la educación media técnico profesional. Para la cohorte en conjunto, se tiene que la remuneración de las mujeres es un 73,2 por ciento de la obtenida por los hombres en la modalidad técnico profesional y 88,9 por ciento en la científico humanista.

La brecha salarial de género en la cohorte técnico profesional se reduce cuando se controla por los años de experiencia laboral, especialmente en los egresados de la enseñanza media y desertores del mismo nivel, que son los grupos que presentan una mayor diferencia de meses acumulados de trabajo entre hombres y mujeres. ${ }^{35}$

Las diferencias salariales de género están también relacionadas con las áreas de especialidad cursadas en la enseñanza media técnico profesional.

${ }^{35}$ Ver cuadro A.11 y cuadro A.12 en Anexo. 
Cuadro 13. SALARIO PROMEDIO DE MUJERES COMO PORCENTAJE DE SALARIO PROMEDIO DE HOMBRES

\begin{tabular}{lll}
\hline & TP & $\mathrm{CH}$ \\
\hline Tituladas universidades & 82,7 & 88,0 \\
Tituladas institutos profesionales & 74,8 & 77,6 \\
Tituladas centros de formación técnica & 69,2 & 75,6 \\
Abandonaron los estudios superiores & 72,8 & 80,5 \\
Egresadas educación media & 69,0 & 74,2 \\
Desertoras de la educación media & 66,1 & 67,0 \\
Total & 73,2 & $88,9^{\#}$ \\
\hline
\end{tabular}

\# Nota: El porcentaje promedio es mayor que el porcentaje de los subgrupos por efectos de composición (las mujeres son más numerosas en los grupos de mayor educación).

Fuente: RECh 2003-2011; SIES 2007-2011; Seguro de Cesantía 2003-2012.

Ello en tanto los hombres son mayoría en los estudios vinculados a los oficios que son mejor pagados en el mercado laboral, mientras que las mujeres son mayoría en áreas de estudios relacionadas con servicios, que son peor pagadas. Pero no sólo eso: la brecha de salarios entre mujeres y hombres es también más pronunciada en las especialidades mejor pagadas. ${ }^{36}$ De esta manera, las pocas mujeres que se especializan en las áreas mejor pagadas obtienen un salario más bajo respecto de los hombres de la misma especialidad. Así, hay retroalimentación de efectos entre el sistema educacional y el mercado laboral. Las mujeres estudian especialidades que son peor pagadas en el mercado laboral, a la vez que el mercado laboral paga menos a las mujeres que se especializan en las áreas mejor pagadas.

En el debate internacional se ha argumentado que la educación vocacional podría generar segmentación a edades tempranas, en tanto encasillaría a estudiantes en trayectorias conducentes a trabajos de menor posición social y remuneración económica. La evidencia aquí presentada muestra que en el caso chileno esta situación tocaría en forma particular a las mujeres, puesto que la brecha salarial que las afecta está muy relacionada con las áreas de estudios que cursan en la enseñanza media técnico profesional. Este efecto parece diluirse a medida que las mujeres realizan estudios más avanzados, congruentes con una nivelación en la adquisición de competencias laborales en los niveles superiores de la enseñanza (ver cuadro 13).

${ }^{36}$ Ver cuadro A.13 en Anexo. 


\subsection{Resultados laborales por tipos de establecimiento en la educación técnico profesional}

¿Hay diferencias en los resultados laborales de los jóvenes de la cohorte proveniente de la enseñanza técnico profesional de acuerdo con el tipo de establecimientos donde realizaron los estudios medios? ¿Hay en particular ventajas de los establecimientos de administración delegada en corporaciones privadas dada su mayor vinculación con los sectores productivos?

La base de datos de registros administrativos unificada que se usa en esta investigación muestra que los jóvenes de la cohorte que estudiaron en corporaciones privadas de administración delegada obtienen en el 2012 un salario que es, en promedio, 12 por ciento más elevado respecto del obtenido en las otras dependencias (cuadro 14). Este resultado obedece a que estos jóvenes logran mayores niveles educacionales y, por consiguiente, remuneraciones más elevadas, pero también a que obtienen salarios promedios más altos a un mismo nivel de escolaridad.

En efecto, un porcentaje notoriamente más elevado de alumnos que estudia en corporaciones privadas accede a la educación superior: 53,6 versus el 44,1 por ciento de establecimientos particulares subvencionados y el 34,6 por ciento de liceos municipales. Estos alumnos también desertan menos: 6,3 versus 15,6 y 17,1 por ciento para las otras dependencias (cuadro A.14 en Anexo).

Así también, los jóvenes que estudiaron en corporaciones privadas obtienen remuneraciones más elevadas entre los titulados en institutos profesionales y centros de formación técnica, los egresados de educación media y entre quienes abandonaron los estudios superiores. La suma de estos grupos representa dos terceras partes de la cohorte técnico profesional con ingresos laborales en el año 2012. Por lo demás, los jóvenes provenientes de corporaciones privadas presentan tasas de ocupación más altas en la mayor parte de los niveles educativos. ${ }^{37}$

Estos resultados pueden deberse a que recibieron una mejor enseñanza en la educación media. Como fue reportado, los alumnos de las corporaciones privadas obtienen mejores resultados promedios en la prueba Simce de segundo medio y en la Prueba de Selección Universitaria en relación con estudiantes de similar nivel socioeconómico $y$ similar rendimiento

\footnotetext{
${ }^{37}$ Cuadro A.15 en Anexo.
} 
académico en la enseñanza básica de las otras dependencias, un resultado sugerente de una mejor enseñanza de contenidos generales en los establecimientos de educación media. No obstante, también hay indicios de que estos jóvenes tienen ventajas iniciales en términos de habilidades y motivaciones, en tanto obtienen mejores resultados en la prueba Simce de octavo básico, al interior de cada segmento socioeconómico.

\section{Cuadro 14. SALARIOS PROMEDIOS SEGÚN TRAYECTORIA EDUCACIONALY DEPENDENCIA DE ENSEÑANZA MEDIA PARA LA COHORTE TÉCNICO PROFESIONAL (MILES DE \$ BRUTOS, 2012)}

\begin{tabular}{lcccc}
\hline & Municipal & $\begin{array}{c}\text { Particular } \\
\text { subven- } \\
\text { cionado }\end{array}$ & $\begin{array}{c}\text { Corporación } \\
\text { delegada }\end{array}$ & Total \\
\hline Titulados universidades & 558,3 & 541,8 & 559,4 & 551,5 \\
Titulados institutos profesionales & 501,6 & 508,9 & 520,7 & 508,3 \\
Titulados centros de formación técnica & 431,1 & 431,5 & 483,7 & 439,3 \\
Estudiantes de educación superior & 330,6 & 347,5 & 347,3 & 340,7 \\
Abandonaron los estudios superiores & 386,5 & 387,2 & 407,5 & 389,9 \\
Egresados educación media & 348,5 & 343,7 & 375,9 & 349,8 \\
Desertores de la educación media & 303,1 & 292,0 & 294,0 & 298,2 \\
Total & 358,7 & 361,8 & 402,0 & 364,9 \\
\hline
\end{tabular}

Fuente: RECh 2003-2011; SIES 2007-2011; Seguro de Cesantía 2003-2012.

\section{Consideraciones finales}

El análisis de las trayectorias educacionales y de la inserción laboral de la cohorte de estudiantes que cursó segundo medio en el año 2003 entrega un conjunto de antecedentes que aportan a un mejor conocimiento de la educación media técnico profesional. El estudio provee también un marco de referencia para investigaciones futuras que indaguen materias más específicas, como los efectos causales de la educación técnico profesional sobre los resultados educacionales y laborales.

El estudio muestra que los estudiantes que optan por la enseñanza media técnica profesional pertenecen a grupos de nivel socioeconómico medio y bajo de la población. El desempeño académico en la educación básica es también un factor relevante en la elección de la modalidad técnico profesional, especialmente en los grupos socioeconómicos medios, donde 
un mal resultado en el Simce de octavo básico hace mucho más probable la asistencia a estudios técnico profesionales. En cambio, los sectores más vulnerables de la población tienden a optar por esta modalidad de estudios con relativa independencia del desempeño en la enseñanza básica.

Los jóvenes de la enseñanza media técnico profesional tienen un rendimiento significativamente más bajo en el Simce de segundo medio y, particularmente, en la Prueba de Selección Universitaria. La comparación se realiza respecto de jóvenes de la enseñanza científico humanista con igual resultado en el Simce de octavo básico, lo que permite controlar por el efecto de variables individuales y del entorno social que influyen sobre los resultados de esas pruebas. Es, por lo tanto, muy probable que estas brechas de rendimiento se originen en diferencias en la enseñanza recibida, un resultado esperado en la Prueba de Selección Universitaria pero no así en el Simce de segundo medio, si se considera que el currículo es común hasta ese nivel.

No obstante lo anterior, los jóvenes que optan por la enseñanza media técnico profesional presentan una menor tasa de deserción respecto de quienes estudian educación general. En particular, para los grupos de nivel socioeconómico bajo y menor rendimiento académico, la tasa de deserción es tres veces más baja en la enseñanza técnico profesional que en la científico humanista.

Más del 40 por ciento de los jóvenes de la cohorte técnico profesional prosigue estudios superiores después de egresar de la enseñanza media; mayoritariamente en institutos profesionales y centros de formación técnica. Este es un contingente numeroso, por lo que este tipo de enseñanza no puede seguir siendo considerado como una fase terminal de estudios. El acceso a la educación superior es diferido en el tiempo y, por lo general, ocurre una vez que los jóvenes están trabajando en el mercado laboral, donde es común la combinación de trabajo y estudios entre los jóvenes que provienen de la enseñanza media técnico profesional.

Cerca del 30 por ciento de la cohorte técnico profesional que ingresa a la educación superior abandona sus estudios, una tasa que duplica a la que presentan los egresados de la enseñanza científico humanista. Alrededor de la mitad de la tasa de deserción en los jóvenes técnico profesionales es atribuible a la condición socioeconómica y al tipo de institución donde realizan los estudios superiores.

Los jóvenes de la cohorte técnico profesional participan más en el mercado laboral que sus contrapartes de la educación científico humanista. 
El resultado rige para todas las trayectorias educacionales, distinguiéndose dos tipos de situaciones: para los titulados de la educación superior el resultado es probable reflejo de que el grupo ya trabajaba cuando estudiaba y no está buscando empleo por primera vez; para egresados de enseñanza media y otros niveles educativos reflejaría, también, diferencias en la dotación de competencias laborales y en la predisposición hacia el trabajo remunerado.

Un conjunto de los egresados de la educación media técnico profesional obtiene un buen nivel de remuneraciones en el mercado del trabajo en relación con sus respectivos grupos de referencia. En particular, éste es el caso de aquellos que siguieron estudios posteriores en centros de formación técnica e institutos profesionales, así como de los hombres que egresan de la enseñanza media y que se especializaron en oficios industriales.

Sin embargo, las mujeres que estudian enseñanza media técnico profesional reciben un mayor castigo salarial en el mercado del trabajo, especialmente aquellas que no prosiguen estudios superiores. En parte ello ocurre porque muchas de ellas se especializan en áreas de estudio que son mal remuneradas en el mercado laboral, como son las relacionadas con los servicios. Por otra parte, las mujeres que estudian en las áreas mejor pagadas presentan las mayores brechas salariales respecto de los hombres de su misma especialidad. Hay así retroalimentación entre el sistema educativo y el mercado laboral, que redunda en bajos salarios para este grupo.

Quienes estudiaron educación técnico profesional en establecimientos de corporaciones privadas obtienen mayores salarios que quienes asistieron a establecimientos municipales o particulares subvencionados. Los primeros logran niveles de escolaridad más altos, a la vez que obtienen mayores remuneraciones a igual nivel de escolaridad. Estos resultados reflejarían, por una parte, una mejor enseñanza, como se constata en los resultados en el Simce de segundo medio y en la Prueba de Selección Universitaria, después de controlar por factores individuales y del entorno social, y, por otra, probables ventajas en habilidades o motivaciones iniciales, según se desprende de los resultados del Simce de octavo básico. EP 


\section{REFERENCIAS}

Bassi, Marina, Matías Busso, Sergio Urzúa \& Jaime Vargas. 2012. Desconectados. Habilidades, educación y empleo en América Latina. Washington, DC: Banco Interamericano de Desarrollo.

Bravo, David. 1999. Evaluación del marco institucional de la educación media técnico profesional. Santiago: Departamento de Economía Universidad de Chile.

Brunello, Giorgio \& Danielle Checchi. 2007. "Does School Tracking Affect Equality of Opportunity?”. Economic Policy 22: 781-861.

Bucarey, Alonso \& Sergio Urzúa. 2013a. "El retorno económico de la educación media técnico profesional en Chile”. Estudios Públicos 129: 1-48.

2013b. "The Economic Return to Vocational High Schools in Chile". Mimeo, University of Maryland.

Cáceres, Carlos \& Eugenio Bobenrieth. 1993. "Determinantes del salario de los egresados de la educación media técnico profesional en Chile". Cuadernos de Economía 89: 111-129.

1994. "Un modelo de elección de liceos de enseñanza media". Cuadernos de Economía 92: 27-44.

Centro de Microdatos. 2009. "Encuesta de seguimiento de egresados de la educación media técnico profesional. Informe final". Santiago: Universidad de Chile.

Cunha, Flavio, James Heckman, Lance Lochner \& Dimitriv Masterov. 2005. "Interpreting the Evidence on Life Cycle Skill Formation". NBER Working Paper 11331. Accedido el 28 abril de 2014. http://www.nber.org/papers/ w11331.

De Iruarrizaga, Francisca. 2009. "Dos miradas a la educación media en Chile". Tesis magíster en economía. Instituto de Economía, Pontificia Universidad Católica de Chile.

Espinoza, Óscar. 2011. "La implementación de la reforma curricular en la educación media técnico profesional: evaluación y proyecciones". Santiago: PIEE, Universidad Academia de Humanismo Cristiano. Accedido el 28 abril de 2014. http://www.cie-ucinf.cl/download/libros_del_cie/La-implementacion-ReformaCurricular-Educacion-Media-Tecnico-Profesional-2.pdf.

Farías, Mauricio. 2013. "Effects of Early Career Decisions on Future Opportunities: The Case of Vocational Education in Chile". PhD diss., Stanford University.

Farías, Mauricio \& Rafael Carrasco. 2012. "Diferencias en resultados académicos entre la educación media técnico profesional y humanista científica en Chile". Calidad en la Educación 36: 87-121.

Farías, Mauricio \& María Paola Sevilla. 2012. "Efectividad de la enseñanza media técnico profesional en la persistencia y rendimiento en la educación técnica superior". Documento de trabajo. Ministerio de Educación.

Hanushek, Eric, Lugder Woessmann \& Lei Zhang. 2011. "General Education, Vocational Education and Labor Market Outcomes Over the Life Cycle". 
NBER Working Paper 17504. Accedido el 28 abril de 2014. http://www.nber. org/papers/w17504.

Larrañaga, Osvaldo, Gustavo Cabezas \& Francisca Dussaillant. 2014. "Estudio de la educación media técnico profesional”. Santiago: PNUD. Accedido el 28 abril de 2014. www.pnud.cl.

Malamud, Ofer \& Cristian Pop-Eleches. 2008. "General Education versus Vocational Training. Evidence from an Economy in Transition". NBER Working Paper 14155. Accedido el 28 abril 2014. http://www.nber.org/papers/w14155.

Meer, Jonathan. 2005. "Evidence on the Returns to Secondary Vocational Education”. SIEPR Discussion Paper n ${ }^{\circ}$ 04-14. Stanford University.

Ministerio de Educación de Chile. 2005. "Contenidos fundamentales y objetivos mínimos obligatorios de la educación media. Actualización 2005”. Santiago.

2009. "Bases para una política de formación técnico profesional en Chile. Comisión para el estudio de la formación técnico profesional en Chile. Informe Ejecutivo". Santiago.

2011. "Educación media técnico profesional en Chile. Antecedentes y claves de diagnóstico". Centro de Estudios. Santiago.

Miranda, Martín. 2003. “Transformación de la educación media técnico profesional". En Políticas educacionales del cambio de siglo, editado por Cristián Cox. Santiago: Editorial Universitaria.

OECD. 2009. Learning for Jobs. OECD Reviews of Vocational Education and Training. Chile: a First Report. París.

Ortiz, Iván. 2009. “¿Es relevante la educación media técnico profesional”. Persona y Sociedad 23: 99-115.

Sahlberg, Pasi. 2007. "Secondary Education in OECD Countries. Common Challenges and Differing Solutions". European Training Foundation. Accedido el 28 abril de 2014. http://etf.europa.eu/webatt.nsf/0/C12578310056925BC125 73850034415B/\$file/NOTE78HD6G.pdf.

Sepúlveda, Leandro, Pamela Ugalde \& Fabiola Campos. 2010. "La enseñanza media técnico profesional en Chile. Orientaciones actuales desde la perspectiva de sus actores". Santiago: CIDE, Universidad Alberto Hurtado. 
ANEXO

Cuadro A.1. DISTRIBUCIÓN DE LA COHORTE AUXILIAR SEGÚN NIVEL SOCIOECONÓMICO Y QUINTILES RESULTADOS SIMCE DE OCTAVO BÁSICO (\%)

\begin{tabular}{ccccccc}
\hline Quintiles Simce & \multicolumn{7}{c}{ Nivel socioeconómico } \\
\hline & 1 & 2 & 3 & 4 & 5 & Total \\
2 & 8,3 & 5,5 & 3,3 & 2,6 & 1,6 & 21,4 \\
3 & 6,7 & 5,1 & 3,5 & 3,3 & 2,3 & 20,9 \\
4 & 5,2 & 4,3 & 3,5 & 3,8 & 3,4 & 20,2 \\
5 & 3,5 & 3,3 & 3,1 & 4,3 & 5,0 & 19,3 \\
total & 1,7 & 1,9 & 2,1 & 4,0 & 8,6 & 18,3 \\
& 25,5 & 20,1 & 15,4 & 18,0 & 20,9 & 100,0 \\
\hline
\end{tabular}

Fuente: RECh 2003 a 2011; Simce 2004 octavo básico, cohorte auxiliar.

Cuadro A.2. REGRESIÓN PROBIT PARA PROBABILIDAD DE ESTUDIAR EDUC. MEDIA TP

\begin{tabular}{|c|c|c|c|c|}
\hline Probit: estudiar TP/ & $\mathrm{d} 1$ & $d 2$ & d3 & d4 \\
\hline \multicolumn{5}{|l|}{ ensenanza media } \\
\hline Mujer & $-0.039^{* * *}$ & $-0.043^{* * *}$ & $-0.043^{* * *}$ & $-0.024^{* * *}$ \\
\hline Nivel socioeconómico 2 & $-0.013^{* *}$ & $-0,002$ & $-0,005$ & 0.007 \\
\hline Nivel socioeconómico 3 & $-0.072^{\star \star \star}$ & $-0.054^{* * *}$ & $-0.063^{\star * *}$ & $-0.032^{\star *}$ \\
\hline Nivel socioeconómico 4 & $-0.212^{* * *}$ & $-0.171^{* \star *}$ & $-0.182^{* * *}$ & $-0.117^{* * *}$ \\
\hline Nivel socioeconómico 5 & $-0.438^{* * *}$ & $-0.387^{* * *}$ & $-0.397^{* * *}$ & $-0.317^{* * *}$ \\
\hline Simce octavo quintil 2 & & $-0,008$ & $-0,007$ & 0.014 \\
\hline Simce octavo quintil 3 & & $-0.039^{* \star *}$ & $-0.037^{\star \star \star}$ & 0.008 \\
\hline Simce octavo quintil 4 & & $-0.106^{* * *}$ & $-0.105^{* * *}$ & -0.034 \\
\hline Simce octavo quintil 5 & & $-0.235^{\star \star *}$ & $-0.236^{* * *}$ & $-0.141^{* * *}$ \\
\hline Región 1 & & & $-0,001$ & $0.038^{* * *}$ \\
\hline Región 2 & & & $-0,002$ & $0.016^{* * *}$ \\
\hline Región 3 & & & $0.116^{\star \star *}$ & $0.138^{* * *}$ \\
\hline Región 4 & & & $-0.133^{\star * *}$ & $-0.136^{* * *}$ \\
\hline Región 5 & & & $-0.079^{* * *}$ & $-0.079^{* * *}$ \\
\hline Región 6 & & & $-0.150^{* * *}$ & $-0.167^{* \star *}$ \\
\hline Región 7 & & & 0,01 & -0.006 \\
\hline Región 8 & & & $-0.077^{\star \star *}$ & $-0.077^{\star * *}$ \\
\hline Región 9 & & & $-0.016^{\star *}$ & $-0.017^{\star * *}$ \\
\hline
\end{tabular}


Región 10

Región 11

Región 12

Padres esperan estudios en

IP o CFT

Padres esperan estudios universitarios

\begin{tabular}{ccccc} 
r2_p & 0,113 & 0,135 & 0,144 & 0,19 \\
$p$ & 0 & 0 & 0 & 0 \\
$\|$ & $-91636,207$ & $-89348,35$ & $-88392,295$ & $-83688,527$ \\
$N$ & 153354 & 153354 & 153354 & 153354 \\
\hline
\end{tabular}

Nota: Los asteriscos señalan significancia estadistica al $1 \%\left(^{* * *}\right) ; 5 \%\left(^{* *}\right)$ y $10 \%\left({ }^{*}\right)$. Los coeficientes son efectos marginales. Las variables de referencias respectivos son: hombres; nivel socioeconómico 1; Simce octavo quintil 1; Región Metropolitana; padres que esperan que hijos realicen estudios medios.

Fuente: RECh 2003-2011; Simce 2003.

Cuadro A.3. REGRESIÓN PROBIT, PADRES QUE ESPERAN QUE HIJOS CONTINÚEN ESTUDIOS SUPERIORES

\begin{tabular}{lc}
\hline Mujer dummy & $0.048^{* * *}$ \\
Quintil simce 2 & $0.071^{\star \star *}$ \\
Quintil simce 3 & $0.126^{* * *}$ \\
Quintil simce 4 & $0.175^{* \star *}$ \\
Quintil simce 5 & $0.229^{* * *}$ \\
SES 2 & $0.084^{* * *}$ \\
SES 3 & $0.167^{* * *}$ \\
SES 4 & $0.239^{* * *}$ \\
SES 5 & $0.318^{* * *}$ \\
r2_P & \\
P & 0,218 \\
LI & 0 \\
$\mathrm{~N}$ & $-87944,13$ \\
\hline
\end{tabular}

Nota: Los asteriscos señalan significancia estadistica al $1 \%\left(^{(* *)}\right) ; 5 \%\left(^{* *}\right)$ y $10 \%\left({ }^{*}\right)$.

Fuente: RECh 2003-2011; Simce 2004, cohorte auxiliar. 
Cuadro A.4. DISTRIBUCIÓN DE LA COHORTE CH SEGÚN NIVEL SOCIOECONÓMICO (\%)

\begin{tabular}{lrrrrrr}
\hline & \multicolumn{7}{c}{ Nivel socioeconómico } \\
\hline Municipal & 1 & 2 & 3 & 4 & 5 & Total \\
Particular subvencionado & 26,1 & 16,7 & 17,6 & 27,1 & 12,4 & 100 \\
Particular pagado & 9,2 & 9,1 & 14,6 & 37,5 & 29,6 & 100 \\
Total & 0,9 & 1,2 & 2,8 & 19,2 & 76,0 & 100 \\
& 16,0 & 11,6 & 14,5 & 30,3 & 27,6 & 100 \\
\hline
\end{tabular}

Fuente: RECh 2003-2011; Simce 2003. Cuadro A.5. REGRESIONES PARA RESULTADOS SIMCE DE SEGUNDO MEDIO
(Cohorte auxiliar)

\begin{tabular}{|c|c|c|c|c|c|c|}
\hline & \multicolumn{3}{|c|}{ Toda la cohorte } & \multicolumn{3}{|c|}{ Alumnos en técnico profesional } \\
\hline & (1) & $(2)$ & (3) & (1) & (2) & (3) \\
\hline Simce 8vo & $0.97^{* * *}$ & $0.98^{* * *}$ & $0.94^{* * *}$ & $0.86^{* * *}$ & $0.86^{* * *}$ & $0.85^{* * *}$ \\
\hline TP dummy & & $23.1^{\star \star \star}$ & $19.0^{\star \star \star}$ & & & \\
\hline TP*simce 8 & & $-0.11^{* * *}$ & $-0.09^{* * *}$ & & & \\
\hline Quintil SES 2 & & & $1.24^{* * *}$ & & $1.18^{* *}$ & $0.96^{* *}$ \\
\hline Quintil SES 3 & & & $2.22^{* * *}$ & & $1.34^{\star \star \star}$ & $0.88^{*}$ \\
\hline Quintil SES 4 & & & $6.16^{* \star \star}$ & & $4.22^{\star * *}$ & $3.54^{\star * *}$ \\
\hline Quintil SES 5 & & & $12.9^{* \star *}$ & & $6.93^{* * *}$ & $6.12^{* \star \star}$ \\
\hline part subv & & & & & & $3.22^{* * *}$ \\
\hline Corporación & & & & & & $8.25^{\star \star *}$ \\
\hline _cons & $5.49^{* * *}$ & $3.41^{* * *}$ & $7.36^{* * *}$ & $26.5^{* * *}$ & $26.2^{* * *}$ & $26.0^{* * *}$ \\
\hline r2 & 0,68 & 0,68 & 0,69 & 0,58 & 0,58 & 0,58 \\
\hline $\mathrm{P}$ & 0 & 0 & 0 & 0 & 0 & 0 \\
\hline N & 159094 & 159094 & 159094 & 65596 & 65596 & 65596 \\
\hline
\end{tabular}

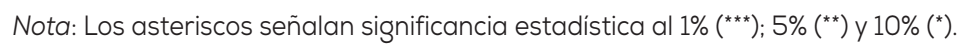

Fuente: RECh 2003-2011; Simce 2004 y 2006. 
Cuadro A.6. REGRESIONES RESULTADOS PRUEBA SELECCIÓN UNIVERSITARIA (Cohorte auxiliar)

\begin{tabular}{lcccccc}
\hline & \multicolumn{3}{c}{ Toda la cohorte } & \multicolumn{3}{c}{ Alumnos en técnico profesional ${ }^{*}$} \\
\hline & $(1)$ & $(2)$ & $(3)$ & $(1)$ & $(2)$ & $(3)$ \\
Simce 8vo & $1.89^{* * *}$ & $1.87^{* *}$ & $1.75^{* * *}$ & $1.45^{* * *}$ & $1.43^{* * *}$ & $1.42^{* * *}$ \\
TP dummy & & $61.5^{* * *}$ & $49.6^{* * *}$ & & & \\
TP*simce 8 & & $-0.41^{* * *}$ & $-0.33^{* * *}$ & & & \\
Quintil SES 2 & & & $4.93^{* * *}$ & $2.56^{* *}$ & $1.96^{*}$ & $2.19^{*}$ \\
Quintil SES 3 & & & $8.07^{* * *}$ & $4.61^{* * *}$ & $3.54^{* * *}$ & $4.29^{* * *}$ \\
Quintil SES 4 & & & $20.5^{* * *}$ & $12.9^{* * *}$ & $11.4^{* * *}$ & $12.1^{* * *}$ \\
Quintil SES 5 & & & $44.2^{* * *}$ & $20.7^{* * *}$ & $18.8^{* * *}$ & $19.4^{* * *}$ \\
Part. subv. & & & & & $8.99^{* * *}$ & $12.1^{* * *}$ \\
Corporación & & & & & $13.6^{* * *}$ & $15.8^{* * *}$ \\
_cons & $-9.43^{* * *}$ & $9.82^{* * *}$ & $17.2^{* * *}$ & $71.3^{* * *}$ & $68.5^{* * *}$ & $66.5^{* * *}$ \\
r2 & 0,63 & 0,68 & 0,69 & 0,52 & 0,53 & 0,54 \\
P & 0,0 & 0,0 & 0,0 & 0,0 & 0,0 & 0,0 \\
N & 118295 & 118295 & 118295 & 37511 & 37511 & 37511 \\
\hline \hline
\end{tabular}

Nota: Los asteriscos señalan significancia estadistica al $1 \%\left({ }^{* * *}\right) ; 5 \%\left({ }^{* *}\right)$ y $10 \%\left({ }^{*}\right)$.

Fuente: RECh 2003-2011; Simce 2004 y PSU 2009.

Cuadro A.7. REGRESIONES PROBIT DE DESERCIÓN EN LA EDUCACIÓN MEDIA (Cohorte auxiliar)

\begin{tabular}{|c|c|c|c|}
\hline & Todos & $\mathrm{CH}$ & TP \\
\hline TP dummy & $-0.056^{* * *}$ & & \\
\hline Mujer dummy & $-0.035^{* * *}$ & $-0.044^{* \star *}$ & $-0.016^{* * *}$ \\
\hline Quintil simce 2 & $-0.033^{* * *}$ & $-0.032^{* * *}$ & $-0.033^{* * *}$ \\
\hline Quintil simce 3 & $-0.057^{\star \star *}$ & $-0.055^{\star \star \star}$ & $-0.050^{* * *}$ \\
\hline Quintil simce 4 & $-0.076^{* * *}$ & $-0.076^{* * *}$ & $-0.064^{* * *}$ \\
\hline Quintil simce 5 & $-0.100^{\star * \star}$ & $-0.114^{* * *}$ & $-0.066^{\star \star \star}$ \\
\hline SES 2 & $-0.010^{\star * \star}$ & $-0.011^{\star * \star}$ & $-0.007^{\star}$ \\
\hline SES 3 & $-0.016^{\star \star \star}$ & $-0.018^{* \star *}$ & $-0.010^{\star \star \star}$ \\
\hline SES 4 & $-0.044^{* \star *}$ & $-0.046^{\star * *}$ & $-0.029^{* * *}$ \\
\hline SES 5 & $-0.065^{* * *}$ & $-0.065^{* * *}$ & $-0.032^{* * *}$ \\
\hline Part. subv. & & & $-0.010^{* * *}$ \\
\hline Corporaciones & & & $-0.025^{\star * *}$ \\
\hline
\end{tabular}




\begin{tabular}{lccc} 
Part subv. & & $-0.008^{* * *}$ & \\
Part pagado & & $-0.039^{* * *}$ & \\
r2_p & 0,123 & 0,185 & 0,044 \\
P & 0 & 0 & 0 \\
LI & $-43000,962$ & $-24169,748$ & $-17627,507$ \\
N & 159527 & 92858 & 65569 \\
\hline
\end{tabular}

Nota: Los asteriscos señalan significancia estadistica al $1 \%\left(^{* * *}\right) ; 5 \%\left(\left(^{* \star}\right)\right.$ y $10 \%\left({ }^{*}\right)$. Fuente: RECh 2003-2011; Simce 2004.

Cuadro A.8. REGRESIÓN PROBIT PARA DESERCIÓN EN EDUCACIÓN SUPERIOR

\begin{tabular}{|c|c|c|c|c|c|c|c|}
\hline & \multicolumn{3}{|c|}{ Toda la cohorte } & \multicolumn{4}{|c|}{ Alumnos provenientes de TP } \\
\hline & (1) & (2) & (3) & (1) & (2) & (3) & (4) \\
\hline $\mathrm{TP}=1$ & $0.061^{* \star *}$ & $0.046^{* \star *}$ & $0.028^{* * *}$ & & & & \\
\hline Mujer = 1 & & $-0.027^{\star \star *}$ & $-0.025^{\star * *}$ & $-0.045^{\star \star \star}$ & $-0.043^{* * *}$ & $-0.047^{* \star *}$ & $-0.045^{\star * *}$ \\
\hline SES 2 & & 0,007 & 0,009 & 0,005 & 0,006 & 0,006 & 0,005 \\
\hline SES 3 & & $0.008^{*}$ & $0.014^{\star * *}$ & 0,013 & $0.018^{*}$ & $0.018^{*}$ & $0.017^{*}$ \\
\hline SES 4 & & $-0.012^{\star \star \star}$ & 0,001 & $-0,006$ & 0,005 & 0,008 & 0,008 \\
\hline SES 5 & & $-0.036^{* \star *}$ & $-0.013^{\star \star *}$ & $-0,014$ & 0 & 0 & 0,001 \\
\hline $\mathrm{IP}=1$ & & & $-0.031^{\star * *}$ & $-0.049^{* \star *}$ & $-0.048^{* * *}$ & $-0.061^{\star \star \star}$ & \\
\hline Uni $=1$ & & & $-0.097^{\star \star *}$ & $-0.105^{\star \star *}$ & $-0.106^{* \star *}$ & $-0.119^{* \star *}$ & \\
\hline $\begin{array}{l}\text { Trabaj y } \\
\text { estud= } 1\end{array}$ & & & & & & $-0.069^{* * *}$ & $-0.068^{* * *}$ \\
\hline Uni/sin acre & & & & & & & $-0.105^{\star \star *}$ \\
\hline Uni/2-3 acre & & & & & & & $-0.102^{\star \star *}$ \\
\hline Uni/4-5 acre & & & & & & & $-0.150^{* * *}$ \\
\hline Uni/6-7 acre & & & & & & & $-0.142^{* * *}$ \\
\hline $\mathrm{IP} / \sin$ acre & & & & & & & $-0.102^{* * *}$ \\
\hline IP/2-3 acre & & & & & & & $-0.048^{* * *}$ \\
\hline IP/4-5 acre & & & & & & & $-0.083^{* * *}$ \\
\hline IP/6-7 acre & & & & & & & $-0.094^{* * *}$ \\
\hline CFT/2-3 acre & & & & & & & -0.016 \\
\hline CFT/4-5 acre & & & & & & & $-0.041^{*}$ \\
\hline CFT/6-7 acre & & & & & & & $-0.091^{\star * *}$ \\
\hline$P$ & 0 & 0 & 0 & 0 & 0 & 0 & 0 \\
\hline LI & $-32556,4$ & $-32390,7$ & $-31866,5$ & $-12278,6$ & $-12328,6$ & $-11879,3$ & $-11763,6$ \\
\hline$N$ & 87240 & 87240 & 87240 & 27790 & 27790 & 26712 & 26712 \\
\hline
\end{tabular}

Nota: Los asteriscos señalan significancia estadistica al $1 \%\left(^{* * *}\right) ; 5 \%\left({ }^{* *}\right)$ y $10 \%\left({ }^{*}\right)$. Fuente: RECh 2003-2011; Simce 2003; SIES 2007 a 2011. 
Cuadro A.9. COHORTE DE EDUCACIÓN TÉCNICO PROFESIONAL POR SECTOR/ ESPECIALIDAD (ÁREA) DE ESTUDIOS

\begin{tabular}{|c|c|c|c|c|}
\hline Sector & Especialidad & Casos & $\%$ en total & $\%$ mujeres \\
\hline Administración & Administración & 17.798 & 17,6 & 60,8 \\
\hline Administración & Contabilidad & 11.166 & 11,1 & 58,1 \\
\hline Administración & Secretariado & 6.101 & 6,0 & 93,1 \\
\hline Administración & Ventas & 4.180 & 4,1 & 60,9 \\
\hline Metalmecánico & Mecánica industrial & 3.645 & 3,6 & 3,2 \\
\hline Metalmecánico & Construcciones metálicas & 2.620 & 2,6 & 3,5 \\
\hline Metalmecánico & Mecánica automotriz & 5.658 & 5,6 & 2,3 \\
\hline Electricidad & Electricidad & 5.684 & 5,6 & 3,9 \\
\hline Electricidad & Electrónica & 5.162 & 5,1 & 6,0 \\
\hline Electricidad & Telecomunicaciones & 1.656 & 1,6 & 28,2 \\
\hline Construcción & & 5.964 & 5,9 & 15,5 \\
\hline Minero & & 836 & 0,8 & 25,0 \\
\hline Gráfica & & 1.342 & 1,3 & 32,6 \\
\hline Químico & & 1.000 & 1,0 & 66,0 \\
\hline Confección & & 1.792 & 1,8 & 95,3 \\
\hline Alimentación & & 8.636 & 8,6 & 69,7 \\
\hline Programas sociales & & 7.813 & 7,7 & 94,0 \\
\hline Hoteleria y turismo & & 2.485 & 2,5 & 74,9 \\
\hline Maderero & & 2.179 & 2,2 & 21,8 \\
\hline Agropecuario & & 3.828 & 3,8 & 30,5 \\
\hline Marítimo & & 1.460 & 1,4 & 36,0 \\
\hline Total & & 101.005 & 100,0 & 47,7 \\
\hline
\end{tabular}

Fuente: RECH 2003-2011; SIES 2007-2011; Seguro de Cesantía 2003-2012. 


\section{Cuadro A.10. REGRESIONES DE SALARIOS PARA EGRESADOS TP SIN EDUCACIÓN SUPERIOR}

\begin{tabular}{|c|c|c|c|c|c|}
\hline & M1 & M2 & M3 & M4 & M5 \\
\hline Minero & $250957^{* * *}$ & $250092^{* * *}$ & $250422^{* * *}$ & $250422^{\star * *}$ & $249964^{* * *}$ \\
\hline Gráfica & 13532 & $35939^{* *}$ & $35947^{* *}$ & $35947^{\star *}$ & $33595^{\star \star}$ \\
\hline Químico & 701 & $38604^{* *}$ & $38621^{* *}$ & $38621^{* *}$ & $37544^{* *}$ \\
\hline Confección & $-128469^{* * *}$ & $-37158^{* \star *}$ & $-37590^{* * *}$ & $-37590^{* * *}$ & $-36208^{* * *}$ \\
\hline Alimentación & $-88947^{\star \star \star}$ & $-44206^{\star \star *}$ & $-43953^{\star \star \star}$ & $-43953^{\star * *}$ & $-43329^{\star \star *}$ \\
\hline Programas y proyectos sociales & $-113106^{\star \star \star}$ & $-35241^{* \star *}$ & $-34834^{\star * *}$ & $-34834^{* * *}$ & $-34996^{* * *}$ \\
\hline Hoteleria y turismo & $-84391^{* * *}$ & $-26878^{* *}$ & $-26079^{* *}$ & $-26079^{* *}$ & $-26313^{\star *}$ \\
\hline Maderero & $-47632^{* \star *}$ & $-43699^{* * *}$ & $-43165^{\star \star *}$ & $-43165^{\star \star \star}$ & $-39096^{\star * *}$ \\
\hline Agropecuario & $-37817^{* * *}$ & $-25169^{* * *}$ & $-24943^{* * *}$ & $-24943^{* * *}$ & $-19802^{* *}$ \\
\hline Marítimo & -4964 & 9215 & 9609 & 9609 & 9227 \\
\hline Administración & $-49018^{* * *}$ & -3936 & -3408 & -3408 & -4269 \\
\hline Contabilidad & $-40918^{* * *}$ & -4731 & -4332 & -4332 & -6166 \\
\hline Secretariado & $-85990^{* * *}$ & -6901 & -6545 & -6545 & -6869 \\
\hline Ventas & $-49903^{* \star *}$ & -11243 & -11383 & -11383 & -12041 \\
\hline Mecánica industrial & $111326^{* * *}$ & $91678^{* * *}$ & $90963^{* * *}$ & $90963^{* * *}$ & $90689^{* * *}$ \\
\hline Construcciones metálicas & $27139^{* * *}$ & $16956^{*}$ & $16275^{*}$ & $16275^{*}$ & $16752^{*}$ \\
\hline Mecánica automotriz & $39916^{* \star *}$ & $30460^{\star \star *}$ & $30860^{* * *}$ & $30860^{* \star *}$ & $31934^{* \star *}$ \\
\hline Electricidad & $74350^{* * *}$ & $56950^{* * *}$ & $56798^{* * *}$ & $56798^{* * *}$ & $56297^{\star * *}$ \\
\hline Electrónica & $17315^{*}$ & 7830 & 8653 & 8653 & 6217 \\
\hline Telecomunicaciones & -10894 & 2064 & 2836 & 2836 & 1376 \\
\hline D_mujer & & $-56168^{\star \star *}$ & $-56261^{\star \star *}$ & $-56261^{\star \star \star}$ & $-55535^{* * *}$ \\
\hline Experiencia & & $3532^{* \star *}$ & $3529^{* \star *}$ & $3529^{* \star \star}$ & $3527^{\star \star \star}$ \\
\hline Calidad estab 2 & & & & & $13525^{\star \star \star}$ \\
\hline Calidad estab 3 & & & & & $19298^{* * *}$ \\
\hline Calidad estab 4 & & & & & $31892^{* * *}$ \\
\hline Calidad estab 5 & & & & & $28040^{* * *}$ \\
\hline SES 2 & & & -1804 & -1804 & -3342 \\
\hline SES 3 & & & 3695 & 3695 & 1132 \\
\hline SES 4 & $375896^{* \star *}$ & $194852^{\star \star \star}$ & $194993^{\star * *}$ & $194993^{* * *}$ & $182365^{* * *}$ \\
\hline SES 5 & $250957^{* * *}$ & $250092^{* * *}$ & $250422^{* * *}$ & $250422^{* * *}$ & $249964^{* * *}$ \\
\hline Part. subvencionado & 13532 & $35939^{* *}$ & $35947^{* *}$ & $35947^{* *}$ & $33595^{* *}$ \\
\hline Corp. adm. delegada & 701 & $38604^{* *}$ & $38621^{* *}$ & $38621^{* *}$ & $37544^{* *}$ \\
\hline Cte & $-128469^{* \star *}$ & $-37158^{* * *}$ & $-37590^{* \star *}$ & $-37590^{* * *}$ & $-36208^{* * *}$ \\
\hline r2 & 0,096 & 0,225 & 0,225 & 0,228 & 0,231 \\
\hline$N$ & 24711 & 24711 & 24711 & 24711 & 24711 \\
\hline
\end{tabular}

Nota: Los asteriscos señalan significancia estadistica al $1 \%\left(^{* * *}\right) ; 5 \%\left(^{* *}\right)$ y $10 \%\left({ }^{*}\right)$.

Fuente: RECh 2003-2011; Simce 2003; Seguro de Cesantía 2003 a 2012. 
Cuadro A.11. BRECHAS SALARIALES DE GÉNERO AJUSTADAS POR EXPERIENCIA LABORAL (\%)

\begin{tabular}{lcc}
\hline & TP & $\mathrm{CH}$ \\
\hline Titulados universidades & 86,0 & 88,2 \\
Titulados institutos profesionales & 76,1 & 78,7 \\
Titulados centros de formación técnica & 74,5 & 77,5 \\
Abandonaron los estudios superiores & 77,2 & 83,0 \\
Egresados de la educación media & 78,6 & 80,2 \\
Desertores de la educación media & 83,9 & 83,7 \\
\hline
\end{tabular}

Fuente: RECh 2003-2011; SIES 2007-2011; Seguro de Cesantía 2003-2012.

Cuadro A.12. MESES TRABAJADOS 2003-2011

\begin{tabular}{lcccccc}
\hline & \multicolumn{3}{c}{ TP } & & \multicolumn{2}{c}{$\mathrm{CH}$} \\
\cline { 2 - 3 } \cline { 6 - 7 } & mujeres & hombres & & mujeres & hombres \\
\hline Titulados universidades & 22,1 & 28,9 & & 12,8 & 13 \\
Titulados institutos profesionales & 31,9 & 37,4 & & 20,2 & 23,9 \\
Titulados centros de formación & 26,7 & 37,4 & & 20,9 & 26,1 \\
técnica & & & & & \\
Estudiantes de educación superior & 27,2 & 32,2 & & 9,4 & 10,9 \\
Abandonaron los estudios superiores & 33,1 & 42,7 & & 19 & 25,3 \\
Egresados educación media & 31,3 & 46,9 & & 23,4 & 34,6 \\
Desertores de educación media & 16,1 & 39,7 & & 15,9 & 37,5 \\
\hline
\end{tabular}

Fuente: RECh 2003-2011; SIES 2007-2011; Seguro de Cesantía 2003-2012. 

Cuadro A.13. BRECHA SALARIAL Y ÁREAS DE ESTUDIOS PARA MUJERES EGRESADAS DE LA EDUCACIÓN TÉCNICO PROFESIONAL SIN ESTUDIOS SUPERIORES (ÁREAS ORDENADAS SEGÚN NIVEL DE REMUNERACIÓN PROMEDIO)

\begin{tabular}{|c|c|c|c|}
\hline Área de estudios & $\begin{array}{l}\text { \% mujeres en } \\
\text { área estudios }\end{array}$ & $\begin{array}{c}\text { Salario mujer } \\
\text { como \% } \\
\text { salario hombre }\end{array}$ & $\begin{array}{c}\text { Tasa de } \\
\text { ocupación } \\
\text { mujeres }\end{array}$ \\
\hline Minero & 14,9 & 68,2 & 63,1 \\
\hline Mecánica industrial & 1,7 & 55,7 & 44,4 \\
\hline Electricidad & 3,5 & 62,1 & 63,9 \\
\hline Químico & 66,3 & 72,0 & 54,8 \\
\hline Construcciones metálicas & 1,7 & 63,8 & 69,4 \\
\hline Mecánica automotriz & 1,4 & 75,3 & 56,1 \\
\hline Electrónica & 4,9 & 81,3 & 64,8 \\
\hline Gráfica & 29,4 & 62,9 & 55,8 \\
\hline Maritimo & 26,7 & 70,9 & 67,1 \\
\hline Construcción & 10,7 & 69,1 & 56,5 \\
\hline Telecomunicaciones & 30,0 & 72,2 & 65,7 \\
\hline Contabilidad & 59,9 & 83,4 & 62,8 \\
\hline Maderero & 13,4 & 66,5 & 48,2 \\
\hline Agropecuario & 22,6 & 71,0 & 56,2 \\
\hline Ventas & 62,6 & 81,5 & 68,9 \\
\hline Administración & 60,9 & 80,0 & 63,2 \\
\hline Hoteleria y turismo & 72,3 & 83,7 & 64,1 \\
\hline Secretariado & 92,9 & 78,9 & 65,8 \\
\hline Alimentación & 65,3 & 77,6 & 61,8 \\
\hline Programas y proyectos sociales & 94,2 & 83,9 & 63,4 \\
\hline Confección & 95,8 & 78,9 & 51,8 \\
\hline Total & 42,5 & 72,6 & 62,6 \\
\hline
\end{tabular}

Fuente: RECh 2003-2011; SIES 2007-2011; Seguro de Cesantía 2003-2012. 
Cuadro A.14. TRAYECTORIAS EDUCACIONALES SEGÚN DEPENDENCIA DE ENSEÑANZA MEDIA PARA LA COHORTE TÉCNICO PROFESIONAL (\%)

\begin{tabular}{lcccc}
\hline & Municipal & $\begin{array}{c}\text { Particular } \\
\text { subven- } \\
\text { cionado }\end{array}$ & $\begin{array}{c}\text { Corporación } \\
\text { delegada }\end{array}$ & Total \\
\hline Titulados universidades & 2,2 & 2,7 & 4,1 & 2,6 \\
Titulados institutos profesionales & 2,5 & 3,3 & 4,8 & 3,1 \\
Titulados centros de formación técnica & 2,3 & 2,6 & 3,1 & 2,5 \\
Estudiantes de educación superior & 16,1 & 21,4 & 25,5 & 19,3 \\
Abandonaron los estudios superiores & 11,6 & 14,9 & 16,1 & 13,5 \\
Egresados educación media & 48,3 & 39,6 & 40,0 & 43,8 \\
Desertores de educación media & 17,1 & 15,6 & 6,3 & 15,2 \\
Total & 100,0 & 100,0 & 100,0 & 100,0 \\
\hline
\end{tabular}

Fuente: RECh 2003-2011; SIES 2007-2011; Seguro de Cesantía 2003-2012.

Cuadro A.15. TASA DE OCUPACIÓN SEGÚN TRAYECTORIA EDUCACIONAL Y DEPENDENCIA DE ENSEÑANZA MEDIA PARA LA COHORTE TÉCNICO PROFESIONAL (\%)

\begin{tabular}{lcccc}
\hline & Municipal & $\begin{array}{c}\text { Particular } \\
\text { subven- } \\
\text { cionado }\end{array}$ & $\begin{array}{c}\text { Corporación } \\
\text { delegada }\end{array}$ & total \\
\hline Titulados universidades & 69,0 & 72,0 & 75,2 & 71,4 \\
Titulados institutos profesionales & 76,7 & 82,9 & 77,8 & 79,6 \\
Titulados centros de formación técnica & 70,7 & 73,0 & 75,9 & 72,4 \\
Estudiantes de educación superior & 62,1 & 62,9 & 63,1 & 62,6 \\
Abandonaron los estudios superiores & 76,0 & 77,9 & 80,5 & 77,4 \\
Egresados educación media & 70,7 & 71,7 & 74,7 & 71,5 \\
Desertores de la educación media & 61,7 & 61,0 & 60,8 & 61,4 \\
Total & 68,5 & 69,5 & 72,0 & 69,3 \\
\hline
\end{tabular}

Fuente: RECh 2003-2011; SIES 2007-2011; Seguro de Cesantía 2003-2012. 